\title{
From fractals and probability to Lévy processes and stochastic PDEs
}

\author{
Davar Khoshnevisan
}

In memory of Oded Schramm (1961-2008)

\begin{abstract}
We present a few choice examples that showcase how the topics in the title are deeply interconnected. Although this is mainly a survey article, it is almost entirely self-contained with complete, albeit somewhat stylized, proofs.
\end{abstract}

Mathematics Subject Classification (2000). Primary 28A78, 28A80, 60J30, $60 \mathrm{H} 15$; Secondary 35R60.

Keywords. Fractals, Lévy processes, stochastic partial differential equations.

\section{Introduction}

It has been known for a long time that the theories of fractals and probability are related. The principle aim of this paper is to make a case for the assertion that those theories are in fact inextricably intertwined. And that, frequently, one learns a good deal by adopting this particular point of view.

I aim to make this case by presenting a series of examples via a self-contained flow of ideas that starts with a classical problem about the ternary Cantor set, and progresses to more modern examples from stochastic analysis that are rooted in statistical mechanics. The approach presented here will have some novelty, even for the classical examples.

The only strict prerequisites to reading this article are a modest knowledge of measure-theoretic probability and a little harmonic analysis on compact abelian groups [for §3.5]. But it might help to know also some stochastic-process theory, "hard" harmonic analysis, and fractal analysis.

And now we begin at the beginning, and without further ado.

Research supported in part by a grant from the National Science Foundation grant DMS-0706728. 


\subsection{The Minkowski dimension}

We begin our discussion by reviewing some basic facts from fractals and geometric measure theory; more detail can be found in the excellent books of Falconer [12] and Mattila [35].

One of the simplest notions of dimension is the [upper] Minkowski dimension, or box dimension, of a bounded set $G \subset \mathbf{R}^{d}$.

Recall that a cube $U \subset \mathbf{R}^{d}$ is dyadic of side $2^{-n}$ if it has the form

$$
U:=\left(\frac{j_{1}}{2^{n}}, \frac{j_{1}+1}{2^{n}}\right] \times \cdots \times\left(\frac{j_{d}}{2^{n}}, \frac{j_{d}+1}{2^{n}}\right],
$$

for some $j:=\left(j_{1}, \ldots, j_{d}\right) \in \mathbf{Z}^{d}$. The collection of such cubes is denoted by $\mathcal{D}_{n}$, and $\mathcal{D}:=\cup_{n=-\infty}^{\infty} \mathcal{D}_{n}$ denotes the collection of all dyadic cubes in $\mathbf{R}^{d}$. On a very few occasions I might refer to $b$-adic cubes of side $b^{-n}$, where $b \geq 2$ is an integer; those are defined also as above, but $2^{n}$ is replaced everywhere by $b^{n}$.

The Minkowski dimension of a bounded set $G \subset \mathbf{R}^{d}$ is defined as

$$
\overline{\operatorname{dim}}_{\mathrm{M}} G:=\limsup _{n \rightarrow \infty} \frac{1}{n} \log _{2}\left(\#\left\{U \in \mathcal{D}_{n}: G \cap U \neq \varnothing\right\}\right),
$$

where " $\log _{2}$ " denotes the base-two logarithm. ${ }^{1}$ It is possible to see that $\overline{\operatorname{dim}}_{\mathrm{M}}$ does not depend on the base. That is, if we use $b$-adic cubes in place of dyadic ones and apply $\log _{b}$ in place of $\log _{2}$, then we obtain the same numerical value for $\overline{\operatorname{dim}}_{\mathrm{M}} G$.

Minkowski dimension is also known as the upper Minkowski [or box] dimension. The corresponding lower Minkowski [or box] dimension is defined as

$$
\underline{\operatorname{dim}}_{\mathrm{M}} G:=\liminf _{n \rightarrow \infty} \frac{1}{n} \log _{2}\left(\#\left\{U \in \mathcal{D}_{n}: G \cap U \neq \varnothing\right\}\right) .
$$

Clearly $\underline{\operatorname{dim}}_{\mathrm{M}} G \leq \overline{\operatorname{dim}}_{\mathrm{M}} G$.

Minkowski dimension is easy to use [and frequently easy to compute], but has the drawback that there are countable sets of positive Minkowski dimension. An example is $G:=\{1,1 / 2,1 / 3, \ldots\}$, whose Minkowski dimension is $1 / 2$. Viewed from this perspective, Hausdorff dimension is a more attractive notion of dimension. We describe that notion next.

\subsection{Net measures and Hausdorff dimension}

Given a number $q \in(0, \infty)$ and a set $G \subset \mathbf{R}^{d}$, we can define the quantity $\mathcal{N}_{q}^{n}(G)$ as $\inf \sum_{k=1}^{\infty}\left(\text { side } I_{k}\right)^{q}$, where the infimum is taken over all dyadic cubes $I_{1}, I_{2}, \ldots$ that have side length $\leq 2^{-n}$ and cover $G$ in the sense that $G \subseteq \cup_{k=1}^{\infty} I_{k}$. The $q$-dimensional net measure of $G$ is the monotonic limit,

$$
\mathcal{N}_{q}(G):=\lim _{n \rightarrow \infty} \mathcal{N}_{q}^{n}(G)
$$

\footnotetext{
${ }^{1}$ As far as I know, Minkowski himself did not study this notion of dimension, but he did introduce the closely-related Minkowski content in geometry.
} 
The restriction of $\mathcal{N}_{q}$ to the Borel sets of $\mathbf{R}^{d}$ - still denoted by $\mathcal{N}_{q}$-is a bona fide Borel measure. The Hausdorff dimension $\operatorname{dim}_{\mathrm{H}} G$ of $G \subset \mathbf{R}^{d}$ is then defined unambiguously as

$$
\operatorname{dim}_{\mathrm{H}} G:=\sup \left\{q>0: \mathcal{N}_{q}(G)>0\right\}=\inf \left\{q>0: \mathcal{N}_{q}(G)<\infty\right\} .
$$

It can be shown that Hausdorff dimension has the following regularity property: For all Borel sets $G_{1}, G_{2}, \ldots \subset \mathbf{R}^{d}$,

$$
\operatorname{dim}_{\mathrm{H}}\left(\bigcup_{n=1}^{\infty} G_{n}\right)=\sup _{n \geq 1} \operatorname{dim}_{\mathrm{H}} G_{n} .
$$

In particular, $\operatorname{dim}_{\mathrm{H}} G=0$ whenever $G$ is countable.

If we use $b$-adic cubes in place of dyadic ones, then we obtain net measures that are, to within constant multiples, the same as $\mathcal{N}_{q}$. Thus, Hausdorff dimension does not depend on the base $b$ that is used.

It can be verified that

$$
\operatorname{dim}_{\mathrm{H}} G \leq \underline{\operatorname{dim}}_{\mathrm{M}} G \leq \overline{\operatorname{dim}}_{\mathrm{M}} G .
$$

Either, or both, of these inequalities can be strict.

\subsection{Riesz capacity}

Typically one computes $\operatorname{dim}_{\mathrm{H}} G$ by separately deriving an upper and a lower bound for it. It is not hard to derive an upper bound in many cases: One strives to construct a "good dyadic cover" $\left\{U_{j}^{n}\right\}_{j=1}^{\infty}$ of side length $\leq 2^{-n}$, and then estimate $\mathcal{N}_{q}^{n}(G)$ from the above by $\sum_{k=1}^{\infty}\left(\text { side } U_{k}^{n}\right)^{q}$. And then one tries to find a value of $q$ that ensures that the said sum is bounded uniformly in $n$; thus, $\operatorname{dim}_{\mathrm{H}} G \leq q$ for such a value of $q$.

It is more difficult to obtain lower bounds, since we have to consider all possible covers. As it turns out, there is a potential-theoretic method that is particularly well-suited to obtaining lower bounds for $\operatorname{dim}_{\mathrm{H}} G$ in many cases.

If $\mu$ is a Borel measure on $\mathbf{R}^{d}$ and $q$ is a positive constant, then the $q$ dimensional Riesz energy of $\mu$ is defined as

$$
I_{q}(\mu):=\iint \frac{\mu(\mathrm{d} x) \mu(\mathrm{d} y)}{\|x-y\|^{q}},
$$

where $\|\cdots\|$ denotes the usual euclidean norm. The quantity $I_{q}(\mu)$ might, or might not, be finite. The following result shows us how we can try to find a lower bound for $\operatorname{dim}_{\mathrm{H}} G$. Here and throughout, $\mathcal{P}(\cdots)$ denotes the collection of all Borel probability measures that have compact support and satisfy $\mu(G)=1$.

Theorem 1.1 (Frostman [15]). If there exist $\mu \in \mathcal{P}(G)$ and $q>0$ such that $I_{q}(\mu)<$ $\infty$, then $\operatorname{dim}_{\mathrm{H}} G \geq q$. Conversely, if $I_{q}(\mu)=\infty$ for all $\mu \in \mathcal{P}(G)$, then $\operatorname{dim}_{\mathrm{H}} G \leq q$.

Define the q-dimensional capacity of a Borel set $G \subset \mathbf{R}^{d}$ as

$$
\operatorname{Cap}_{q}(G):=\frac{1}{\inf _{\mu \in \mathcal{P}(G)} I_{q}(\mu)},
$$


where inf $\varnothing:=\infty$ and $1 / \infty:=0$. Frostman's theorem implies that

$$
\operatorname{dim}_{\mathrm{H}} G=\sup \left\{q>0: \operatorname{Cap}_{q}(G)>0\right\}=\inf \left\{q>0: \operatorname{Cap}_{q}(G)=0\right\} .
$$

In particular, "capacity dimension $=$ Hausdorff dimension." I demonstrate the easier - and more useful - half of Theorem 1.1 next.

Half of the proof. We suppose that $I_{q}(\mu)<\infty$ for some $q>0$ and $\mu \in \mathcal{P}(G)$, and prove that $\operatorname{dim}_{\mathrm{H}} G \geq q$, as a consequence.

For all $\epsilon>0$ we can find a dyadic cover $\left\{U_{j}\right\}_{j=1}^{\infty}$ of $G$ such that $\sum_{j=1}^{\infty}\left|\operatorname{side} U_{j}\right|^{q}$ is at most $\mathcal{N}_{q}(G)+\epsilon$. We fix this cover in mind, and use it as follows:

$$
I_{q}(\mu) \geq \sum_{j=1}^{\infty} \iint_{U_{j} \times U_{j}} \frac{\mu(\mathrm{d} x) \mu(\mathrm{d} y)}{\|x-y\|^{q}} \geq \mathrm{const} \cdot \sum_{j=1}^{\infty} \frac{\left[\mu\left(U_{j}\right)\right]^{2}}{\left|\operatorname{side} U_{j}\right|^{q}} .
$$

Since $\left\{U_{j}\right\}_{j=1}^{\infty}$ is a cover for $G$ and $\mu$ is supported on $G$, Jensen's inequality implies that for all positive numbers $a_{1}, a_{2}, \ldots$,

$$
\sum_{j=1}^{\infty} \frac{1}{a_{j}} \mu\left(U_{j}\right) \geq\left[\sum_{j=1}^{\infty} a_{j} \mu\left(U_{j}\right)\right]^{-1} .
$$

We can set $a_{j}:=\mid$ side $\left.U_{j}\right|^{q} / \mu\left(U_{j}\right)$ to find that

$$
I_{q}(\mu) \geq \frac{\text { const }}{\sum_{j=1}^{\infty}\left|\operatorname{side} U_{j}\right|^{q}} \geq \frac{\text { const }}{\mathcal{N}_{q}(G)+\epsilon} .
$$

We let $\epsilon \rightarrow 0$ and then optimize over all $\mu \in \mathcal{P}(G)$ such that $I_{q}(\mu)$ is finite to find that $\mathcal{N}_{q}(G) \geq$ const $\cdot \operatorname{Cap}_{q}(G)>0$. Consequently, positive capacity implies positive net measure, whence the result.

\section{Some instructive examples}

\subsection{The ternary Cantor set}

Every $x \in[0,1]$ can be written as $x=\sum_{j=1}^{\infty} x_{j} 3^{-j}$ where the digits $x_{j}$ are 0,1 , or 2. The ternary Cantor set $\mathrm{C}$ can be viewed as the collection of all points in $[0,1]$ whose ternary digits are in $\{0,2\}$. Ours differs from the more popular definition of $\mathrm{C}$ by at most a countable collection of points; consequently the two definitions lead to the same Hausdorff dimension. Its numerical value is contained within the following famous result of Hausdorff.

Theorem 2.1 (Hausdorff [19]). $\operatorname{dim}_{\mathrm{H}} \mathrm{C}=\log _{3} 2$.

Proof. The upper bound is derived by a standard covering argument, which I omit. Next you will find a proof of the lower bound that highlights some of Theorem 2.1 's deep connections to probability theory: Let $\left\{X_{j}\right\}_{j=1}^{\infty}$ denote a collection of independent random variables, each taking the values 0 or 2 with probability $1 / 2$. Then the $j$ th ternary digit of $X:=\sum_{j=1}^{\infty} X_{j} 3^{-j}$ is $X_{j}$. Clearly, $\mu(A):=\mathrm{P}\{X \in A\}$ 
defines a Borel probability measure on C. $^{2}$ It suffice to prove that $I_{q}(\mu)<\infty$ for all $q<\log _{3} 2$.

We might observe that if $Y$ is independent of $X$ and has the same distribution $\mu$ as $X$, then $I_{q}(\mu)=\mathrm{E}\left(|X-Y|^{-q}\right)$, where $\mathrm{E}$ denotes expectation. Let $Y_{j}$ denote the $j$ th ternary digit of $Y$, and consider

$$
J:=\inf \left\{j \geq 1: X_{j} \neq Y_{j}\right\} .
$$

The triangle inequality shows that $|X-Y| \geq 3^{-J}$, whence $I_{q}(\mu) \leq \mathrm{E}\left(3^{q J}\right)$. Because $\mathrm{P}\{J=j\}=2^{-j}$ for all integers $j \geq 1, \mathrm{E}\left(3^{q J}\right)=\sum_{j=1}^{\infty} 3^{q j} 2^{-j}$ is finite if and only if $q<\log _{3} 2$, and the theorem follows.

\subsection{Non-normal numbers}

We follow Borel [5] and say that a number $x \in(0,1]$ is simply normal in base 2 if

$$
f(x):=\lim _{n \rightarrow \infty} \frac{1}{n} \sum_{j=1}^{n} \mathbf{1}_{\{0\}}\left(x_{j}\right)=\frac{1}{2},
$$

where $x_{j}$ denotes the $j$ th digit in the binary expansion of $x .^{3}$

The celebrated normal-number theorem of Borel [5] asserts that Lebesguealmost all $x \in(0,1]$ are simply normal in base 2 [and a little more, in fact]. Next is Borel's ingenious proof; it is simplified thanks to more than a century of afterthought.

Let $X:=\sum_{j=1}^{\infty} X_{j} 2^{-j}$, where $\left\{X_{j}\right\}_{j=1}^{\infty}$ are independent random variables, each distributed uniformly on $\{0,1\}$. Then one verifies easily that $X$ is distributed uniformly on $[0,1]$; that is, $\mathrm{P}\{X \in A\}$ is the Lebesgue measure of $A \subseteq[0,1]$. By the strong law of large numbers, $\mathrm{P}\left\{X \in \mathrm{N}_{1 / 2}\right\}=1$ where $\mathrm{N}_{1 / 2}$ denotes the collection of all $x \in(0,1]$ that are simply normal in base 2 . We have shown that $\mathrm{N}_{1 / 2}$ has full measure, and this completes the proof!

It turns out that many interesting nonnormal numbers form fractal collections. For instance, choose and fix a number $p \in(0,1)$ and define

$$
\mathrm{N}_{p}:=\{x \in(0,1]: f(x)=p\} .
$$

Thus, the elements of $\mathrm{N}_{p}$ are numbers whose digits have the prescribed asymptotic freqencies $p$ and $1-p$. The following striking example was conjectured originally by I. J. Good and resolved ultimately by H. G. Eggleston [11]. This example highlights some of the connections between fractals, probability theory, and notions from statistical mechanics.

Theorem 2.2 (Eggleston [11]). $\operatorname{dim}_{\mathrm{H}} \mathrm{N}_{p}=H(p)$, where

$$
H(p):=p \log _{2}\left(\frac{1}{p}\right)+(1-p) \log _{2}\left(\frac{1}{1-p}\right) .
$$

\footnotetext{
${ }^{2}$ In fact, $\mu(\bullet)$ is the restriction of the net measure $\mathcal{N}_{\log _{3}(2)}(\bullet)$ to C.

${ }^{3}$ The $x_{j}$ 's are clearly defined uniquely for all but a countable collection of $x \in[0,1]$; as that collection has zero Lebesgue measure, we can safely not worry about it.
} 
Proof. Let $X_{1}, X_{2}, \ldots$ be independent random variables, each taking the values zero and one with respective probabilities $p$ and $1-p$. Then, $X:=\sum_{j=1}^{\infty} X_{j} 2^{-j}$ is a random number in $(0,1]$ that satisfies $\mathrm{P}\left\{X \in \mathrm{N}_{p}\right\}=1$, owing to the strong law of large numbers. Let $\mu:=\mathrm{P} \circ X^{-1}$ denote the distribution of $X$; we have just seen that $\mu$ is a probability measure on $\mathrm{N}_{p}$.

We begin with a direct computation: If $x \in \mathrm{N}_{p}$ is fixed, then

$$
\begin{aligned}
\mathrm{P}\left\{X_{1}=x_{1}, \ldots, X_{n}=x_{n}\right\} & =p^{n f(x ; n)}(1-p)^{n-f(x ; n)} \\
& =2^{-n(H(p)+o(1))} \quad \text { as } n \rightarrow \infty,
\end{aligned}
$$

where $f(x ; n):=\sum_{j=1}^{n} \mathbf{1}_{\{0\}}(x)$. Note that the little-o term in $(2.5)$ is allowed to depend on the point $x$.

Consider the dyadic cube $U_{n}(x):=\left\{y \in(0,1]: y_{1}=x_{1}, \ldots, y_{n}=x_{n}\right\}$. The preceding shows that $\mu\left(U_{n}(x)\right)=2^{-n\{H(p)+o(1)\}}$ for all $x \in \mathrm{N}_{p}$. Since $y \in U_{n}(x)$ if and only if $x \in U_{n}(y)$, it follows fairly easily that $\mu\left(U_{n}(x)\right)=2^{-n\{H(p)+o(1)\}}$ for all $x \in(0,1]$. It is possible to prove that the following hold:

1. $U_{n}(x) \subseteq\left[x-2^{-n}, x+2^{-n}\right]$; and

2. $\left[x-2^{-n-1}, x+2^{-n-1}\right] \subset U_{n}(y) \cup U_{n}(z)$ for some $y, z \in(0,1]$ that might-or might not-be distinct.

A monotonicity argument then shows that for our $\mu \in \mathcal{P}\left(\mathrm{N}_{p}\right)$,

$$
\mu([x-r, x+r])=r^{H(p)+o(1)} \quad \text { as } r \downarrow 0 \text {, for all } x \in(0,1] .
$$

The density theorem of Rogers and Taylor [45] finishes the proof.

\subsection{The range of Brownian motion}

Let $\mathbf{B}:=\{B(t)\}_{t \geq 0}$ denote Brownian motion in $\mathbf{R}^{d}$. That is, $\mathbf{B}$ is a collection of random variables that satisfy the following: (a) $B(0):=0$; (b) $B(t+s)-B(s)$ is independent of $\{B(u)\}_{0 \leq u \leq s}$ for all $s, t \geq 0$; and (c) the coordinates of the random vector $B(t+s)-B(s)$ are independent mean-zero gaussian random variables with variance $t$, regardless of the value of $s \geq 0$ and $t>0$.

A well-known theorem of Wiener states that one can construct $\mathbf{B}$ such that the resulting random function $t \mapsto B(t)$ is almost surely Hölder continuous with any given index $<1 / 2$. In fact, the following limit exists with probability one:

$$
\lim _{\epsilon \rightarrow 0} \sup _{\substack{s \in[0, T] \\ t \in(s, s+\epsilon]}} \frac{|B(t)-B(s)|}{\sqrt{2(t-s) \ln (t-s)}}=1 \quad \text { for all } T>0 .
$$

This is due to Lévy [31] but with a limsup in place of the limit; the present, more elegant, formulation can be found in Orey and Pruitt [39] and Csörgő and Révész [7].

Theorem 2.3 (Lévy [31], Taylor [51]). $\operatorname{dim}_{\mathrm{H}} B[0, b]=\min (d, 2)$ almost surely for all $b>0$. 
Proof. We need to prove only the lower bound; the upper bound follows readily from the Hölder continuity of $W$ and (1.7).

Let us define a Borel measure $\mu$ via

$$
\mu(V):=\int_{0}^{b} \mathbf{1}_{\{B(s) \in V\}} \mathrm{d} s .
$$

I plan to prove that $\mathrm{E}\left(I_{q}(\mu)\right)<\infty$ for every positive $q<\min (d, 2)$.

Note that

$$
\mathrm{E}\left(I_{q}(\mu)\right)=\mathrm{E}\left(\int_{[0, b]^{2}} \frac{\mathrm{d} s \mathrm{~d} t}{\|B(s)-B(t)\|^{q}}\right) .
$$

Elementary properties of gaussian random vectors show that the distribution of $B(t)-B(s)$ is the same as the distribution of $|t-s|^{1 / 2}$ times $B(1)$, and hence

$$
\mathrm{E}\left(I_{q}(\mu)\right)=\int_{[0, b]^{2}} \frac{\mathrm{d} s \mathrm{~d} t}{|s-t|^{q / 2}} \cdot \mathrm{E}\left(\|B(1)\|^{-q}\right) .
$$

Since the $(\mathrm{d} s \times \mathrm{d} t)$-integral is finite iff $q<2$, it suffices to prove that $\mathrm{E}\left(\|B(1)\|^{-q}\right)$ is finite when $q<d$. But direct computation reveals that

$$
\mathrm{E}\left(\|B(1)\|^{-q}\right)=\frac{1}{(2 \pi)^{d / 2}} \int_{\mathbf{R}^{d}}\|z\|^{-q} \mathrm{e}^{-\|z\|^{2} / 2} \mathrm{~d} z,
$$

and the latter integral is finite iff $q<d$. The theorem follows.

\subsection{Fractal percolation}

Mandelbrot [34] has introduced the following random Cantor set in the context of turbulence: ${ }^{4}$ Choose and fix a parameter $p \in(0,1)$, and let $\{Z(I)\}_{I \in \mathcal{D}}$ be an independent, identically-distributed collection of random variables, each taking the values zero and one with respective probabilities $1-p$ and $p$. Define

$$
Q_{n}(p):=\bigcup\left\{I \in \mathcal{D}_{n} \cap(0,1]^{d}: Z(I)=1\right\} \quad \text { for all } n \geq 0 .
$$

And

$$
\Lambda_{n}(p):=\bigcap_{j=0}^{n} Q_{j}(p) \quad \text { for all } n \geq 0 .
$$

In words, in order to construct $\Lambda_{n}(p)$ from $\Lambda_{n-1}(p)$, we consider each dyadic $I \subset$ $\Lambda_{n-1}(p)$ of sidelength $2^{-n}$ and retain it in $\Lambda_{n}(p)$-independently of the otherswith probability $p$. Since $\Lambda_{n}(p) \subseteq \Lambda_{n-1}(p)$, the following "fractal-percolation set" is well defined:

$$
\Lambda_{p}:=\bigcap_{n=0}^{\infty} \Lambda_{n}(p)
$$

Let $N_{n}$ denote the number of all $I \in \mathcal{D}_{n} \cap(0,1]^{d}$ that are in $\Lambda_{n}(p)$. Then, a little thought shows that $\left\{N_{n}\right\}_{n=0}^{\infty}$ is a Galton-Watson branching process with mean offspring distribution $p 2^{d}$. Therefore, the theory of branching processes tells

${ }^{4}$ Mandelbrot refers to this as "random curdling." 
us the following: If $p>2^{-d}$ then $\sigma:=\mathrm{P}\left\{\Lambda_{p} \neq \varnothing\right\}>0$; else, if $p \leq 2^{-d}$ then $\sigma=0 .{ }^{5}$ Thus, from now on we concentrate on the nontrivial case that

$$
p>2^{-d} \text {. }
$$

Note that $\Lambda_{n}(p)$ is a disjoint union of $N_{n}$ dyadic cubes of sidelength $2^{-n}$ each. Therefore, its Lebesgue measure is $N_{n} 2^{-n d}$. A standard computation reveals that $\mathrm{E}\left(N_{n}\right)=p^{n}$; see (2.17) below, for example. Therefore, $\Lambda_{p}$ has zero Lebesgue measure, almost surely.

Theorem 2.4 (Falconer [13], Mauldin and Williams [36]; see also Grimmett [18]). If (2.15) holds, then

$$
\left\|\operatorname{dim}_{\mathrm{H}} \Lambda_{p}\right\|_{L^{\infty}(\mathrm{P})}=\left\|\overline{\operatorname{dim}}_{\mathrm{M}} \Lambda_{p}\right\|_{L^{\infty}(\mathrm{P})}=d-\log _{2}(1 / p),
$$

where " $\log _{2}$ " denotes the logarithm in base 2 .

Proof. Choose and fix two integers $n>k \geq 1$. The probability that a given dyadic interval $I \in \mathcal{D}_{k} \cap(0,1]$ intersects $\Lambda_{n}(p)$ is $p^{k}$, since $I$ intersects $\Lambda_{n}(p)$ if and only if $Z(J)=1$ for all $k$ dyadic cubes that include $I$. Consequently,

$$
\mathrm{P}\left\{I \cap \Lambda_{p} \neq \varnothing\right\} \leq p^{k} .
$$

Let $N_{k}$ denote the number of dyadic cubes $I \in \mathcal{D}_{k}$ that intersect $\Lambda_{p}$. We can sum (2.17) over all $I \in \mathcal{D}_{k}$ to find that $\mathrm{E}\left(N_{k}\right)=\left(2^{d} p\right)^{k}$. Therefore, Chebyshev's inequality implies that for every positive $\lambda<2^{d} p$,

$$
\sum_{k=1}^{\infty} \mathrm{P}\left\{N_{k}>\lambda^{k}\right\} \leq \sum_{k=1}^{\infty} \frac{\left(2^{d} p\right)^{k}}{\lambda^{k}}<\infty .
$$

Since $\lambda \in\left(0,2^{d} p\right)$ is arbitrary, the preceding and the Borel-Cantelli lemma together show that $\operatorname{dim}_{\mathrm{M}} \Lambda_{p} \leq \log _{2}\left(2^{d} p\right)=d-\log _{2}(1 / p)$ almost surely.

In order to prove the remainder of the theorem, we apply an elegant "intersection argument" of Peres [41]. Let $G$ be a nonrandom Borel set in $(0,1]^{d}$. I will prove the following:

Claim A. If $\operatorname{dim}_{\mathrm{H}} G<\log _{2}(1 / p)$, then $\Lambda_{p} \cap G=\varnothing$ almost surely.

We first use this to prove Theorem 2.4, and then establish Claim A.

Consider an independent fractal percolation $\Lambda_{\beta}^{\prime}$. Because $\Lambda_{p} \cap \Lambda_{\beta}^{\prime}$ has the same distribution as $\Lambda_{p \beta}$, we see that if $p \beta 2^{d}>1$ then $\Lambda_{p} \cap \Lambda_{\beta}^{\prime} \neq \varnothing$ with positive probability. We condition on $\Lambda_{p}$ to deduce from Claim A that $\operatorname{dim}_{\mathrm{H}} \Lambda_{p} \geq \log _{2}(1 / \beta)$ with positive probability. Since any positive $1 / \beta<p 2^{d}$ leads to this bound, it follows that $\left\|\operatorname{dim}_{\mathrm{H}} \Lambda_{p}\right\|_{L^{\infty}(\mathrm{P})} \geq \log _{2}\left(2^{d} p\right)=d-\log _{2}(1 / p)$, as needed. Now let us prove Claim A.

${ }^{5}$ In fact, if we define $\mathcal{N}:=\#\left\{I \in \mathcal{D}_{1}: Z(I)=1\right\}$ - that is, $\mathcal{N}$ denotes the number of retained level-1 dyadic cubes-then $\sigma=\mathrm{E}\left(\sigma^{\mathcal{N}}\right)$ by independence. Since $\mathcal{N}$ has a binomial distribution with parameters $2^{d}$ and $p$, the assertion follows from direct computation. 
We fix an arbitrary $\epsilon>0$, and find dyadic cubes $U_{1}, U_{2}, \ldots$ such that: (i) $\left\{U_{j}\right\}_{j=1}^{\infty}$ is a cover for $G$; and (ii) $\sum_{j=1}^{\infty} \mid$ side $\left.U_{j}\right|^{\log _{2}(1 / p)}<\epsilon$. In accord with (2.17),

$$
\mathrm{P}\left\{\Lambda_{p} \cap G \neq \varnothing\right\} \leq \sum_{j=1}^{\infty} \mathrm{P}\left\{\Lambda_{p} \cap U_{j} \neq \varnothing\right\}=\sum_{j=1}^{\infty} p^{\log _{2}\left(1 / \text { side } U_{j}\right)}<\epsilon .
$$

This completes our proof, since $\epsilon>0$ is arbitrary.

Remark 2.5 (Theorem 2.4, revisited). Because $\Lambda_{p}$ has a great deal of self-similarity, Theorem 2.4 can be improved to the following $[13,36]$, which we prove next:

$$
\mathrm{P}\left\{\operatorname{dim}_{\mathrm{H}} \Lambda_{p}=\overline{\operatorname{dim}}_{\mathrm{M}} \Lambda_{p}=d-\log _{2}(1 / p) \mid \Lambda_{p} \neq \varnothing\right\}=1 .
$$

Let us recall (2.15), let $\mathcal{N}$ denote the number of $I \in \mathcal{D}_{1}$ such that $Z(I)=1$, and choose $\lambda \in\left(0, d-\log _{2}(1 / p)\right)$. We can consider the probabilities

$$
\alpha(I):=\mathrm{P}\left\{\operatorname{dim}_{\mathrm{H}}\left(\Lambda_{p} \cap I\right) \leq \lambda, \Lambda_{p} \cap I \neq \varnothing\right\},
$$

defined for all dyadic cubes $I \subset(0,1]^{d}$. We can condition on $\{Z(I)\}_{I \in \mathcal{D}_{1}}$ to find that $\alpha\left((0,1]^{d}\right) \leq \mathrm{E}\left(\prod \alpha(I) ; \mathcal{N} \geq 1\right)$, where the product is over all $I \in \mathcal{D}_{1} \cap(0,1]^{d}$ such that $Z(I)=1$. But $\alpha(I)=\alpha\left((0,1]^{d}\right)$ for every dyadic cube $I \subseteq(0,1]^{d}$. Therefore,

$$
\begin{aligned}
\alpha\left((0,1]^{d}\right) & \left.\leq \mathrm{E}\left(\left[\alpha(0,1]^{d}\right)\right]^{\mathcal{N}} ; \mathcal{N} \geq 1\right) \\
& \leq \alpha\left((0,1]^{d}\right) \cdot \mathrm{P}\{\mathcal{N} \neq 0\} .
\end{aligned}
$$

Theorem 2.4 implies that $\alpha\left((0,1]^{d}\right)<1$. Since $\mathrm{P}\{\mathcal{N} \neq 0\}<1$, the preceding proves that $\alpha\left((0,1]^{d}\right)=0$, which implies (2.20) readily.

\section{Lévy processes}

Lévy processes are a natural family of random processes that "cannot help but produce fractals." I present a very brief introduction to the general theory. The books by Bertoin [3], Jacob [23], and Sato [48] are excellent, and thorough, accounts of Lévy processes.

A Lévy process $\mathbf{X}:=\{X(t)\}_{t \geq 0}$ on $\mathbf{R}^{d}$ is a stochastic process [that is, a collection of random variables] which satisfies the following properties:

1. $X(0)=0$ almost surely;

2. $X(t+s)-X(s)$ is independent of $\{X(u)\}_{0 \leq u \leq s}$ for all $s, t \geq 0$;

3. The distribution of $X(t+s)-X(s)$ does not depend on $s$, for all $s, t \geq 0$; and

4. $t \mapsto X(t)$ is continuous in probability; i.e., $\lim _{s \rightarrow t} \mathrm{P}\{|X(s)-X(t)|>\epsilon\}=0$ for all $t \geq 0$ and $\epsilon>0$.

It turns out that one can always arrange things such that $t \mapsto X(t)$ is rightcontinuous and has left limits at all points [3, p. 13]; in particular, $\mathbf{X}$ can have only discontinuities of the first kind, if it is at all discontinuous. 
According to the Lévy-Khintchine formula (Bertoin [3, Theorem 1, p. 13]), a stochastic process $\mathbf{X}$ is a Lévy process if and only if

$$
\mathrm{E}\left(\mathrm{e}^{i \xi \cdot X(t)}\right)=\mathrm{e}^{-t \Psi(\xi)} \quad \text { for all } \xi \in \mathbf{R}^{d} \text { and } t>0 \text {, }
$$

where $\Psi$ is a negative-definite function - in the sense of Schoenberg [49] - such that $\Psi(0)=0$; see also the monographs by Jacob [23] and Sato [48]. An equivalent statement is this: If $\mu_{t}(A):=\mathrm{P}\{X(t) \in A\}$ defines the distribution of $X(t)$, then $\left\{\mu_{t}\right\}_{t \geq 0}$ is a weakly-continuous convolution-semigroup of probability measures with $\mu_{0}:=\delta_{0}[3$, Chapter 1$]$. Of course, in this case we have $\int_{\mathbf{R}^{d}} \exp (i \xi \cdot x) \mu_{t}(\mathrm{~d} x)=$ $\exp (-t \Psi(\xi))$. In general, we might refer to the function $\Psi$ as the characteristic exponent, or Lévy exponent, of the process $\mathbf{X}$.

Example. It might be good to keep some examples of Lévy processes in mind:

1. If $\Psi(\xi)=c\|\xi\|^{\alpha}$ for some $c>0$, all $\xi \in \mathbf{R}^{d}$, and $\alpha \in(0,2]$, then $\mathbf{X}$ is an isotropic stable process on $\mathbf{R}^{d}$ with index $\alpha$. When $\alpha=2$ and $c=1 / 2, \mathbf{X}$ is a Brownian motion.

2. The Poisson process is a well-known Lévy process on $\mathbf{Z}_{+}:=\{0,1, \ldots\}$; its characteristic exponent is $\Psi(\xi)=\lambda\left(1-\mathrm{e}^{i \xi}\right)$ for $\xi \in \mathbf{R}$ and $\lambda>0$ is its rate.

3. A compensated Poisson process is a Lévy process on $\mathbf{R}$, and its characteristic exponent is $\Psi(\xi)=\lambda\left(1+i \xi-\mathrm{e}^{i \xi}\right)$. If $\mathbf{Y}$ is a rate- $\lambda$ Poisson process on $\mathbf{Z}_{+}$, then $X(t):=Y(t)-\lambda t$ defines a compensated Poisson process with rate $\lambda$.

Perhaps one of the most common features of many interesting fractals is that they have zero Lebesgue measure. The next result is a characterization of all Lévy processes whose range has zero Lebesgue measure; those are Lévy processes that tend to "generate" random fractals. With this in mind, let us define

$$
\kappa(\xi):=\operatorname{Re}\left(\frac{1}{1+\Psi(\xi)}\right) \quad \text { for all } \xi \in \mathbf{R}^{d}
$$

Theorem 3.1 (Kesten [26]; see also Orey [38]). Choose and fix $b>0$. Then the Lebesgue measure of $X[0, b]$ is positive with positive probability if and only if $\kappa \in$ $L^{1}\left(\mathbf{R}^{d}\right)$.

Remark 3.2. It is well known that Brownian motion is "extremal" among all Lévy processes in the sense that $|\Psi(\xi)| \leq$ const $\cdot\left(1+\|\xi\|^{2}\right)$. Therefore, $\kappa(\xi) \geq$ const $/\left(1+\|\xi\|^{2}\right)$; see Bochner [4, eq. (3.4.14), p. 67], for instance. As a result, we can deduce the following theorem of Lévy [31, Théorèm 53, p. 256]: If $d \geq 2$ then $X[0, b]$ has zero Lebesgue measure almost surely.

Remark 3.3. The passing reference to fractals should not, and can not, be taken too seriously. For example, if $\mathbf{X}$ is a Poisson process on the line with rate $\lambda>0$, then $\Psi(\xi)=\lambda\left(1-\mathrm{e}^{i \xi}\right)$, and $\kappa \notin L^{1}(\mathbf{R})$. Thus, Theorem 3.1 verifies the elementary fact that the range of $\mathbf{X}$ has zero Lebesgue measure. That range is $\mathbf{Z}_{+}$, which is clearly not an interesting fractal. 
It is more difficult to compute the Hausdorff dimension of $X[0, b]$ than to decide when $X[0, b]$ has positive Lebesgue measure. In order to describe the Hausdorff dimension of $X[0, b]$ we consider the "Cauchy transform" $W$ of $\kappa$,

$$
W(r):=\int_{\mathbf{R}^{d}} \frac{\kappa(\xi / r)}{\prod_{j=1}^{d}\left(1+\xi_{j}^{2}\right)} \mathrm{d} \xi \quad \text { for all } r>0 .
$$

Theorem 3.4 (Khoshnevisan and Xiao [28]). For all $b>0$, the following holds with probability one:

$$
\operatorname{dim}_{\mathrm{H}} X[0, b]=\underline{\operatorname{dim}}_{\mathrm{M}} X[0, b]=\liminf _{r \rightarrow 0} \frac{\log W(r)}{\log r} .
$$

Remark 3.5. One can also prove that almost surely,

$$
\operatorname{dim}_{\mathrm{P}} X[0, b]=\overline{\operatorname{dim}}_{\mathrm{M}} X[0, b]=\limsup _{r \rightarrow 0} \frac{\log W(r)}{\log r},
$$

where $\operatorname{dim}_{\mathrm{P}}$ denotes the packing dimension [28].

Example. It is possible to check directly that if $\mathbf{X}$ is Brownian motion on $\mathbf{R}^{d}$, then $\operatorname{dim}_{\mathrm{H}} X[0, b]=\min (d, 2)$ almost surely. This agrees with Theorem 2.3.

\subsection{Subordinators: An example}

A real-valued Lévy process $\mathbf{X}$ is a subordinator if $t \mapsto X(t)$ is almost surely increasing and everywhere nonnegative. We have seen already that one can characterize a subordinator $\mathbf{X}$ by its characteristic exponent $\Psi$. But it is sometimes simpler to consider its Laplace exponent $\Phi: \mathbf{R}_{+} \rightarrow \mathbf{R}_{+}$[2]; the defining feature of $\Phi$ is that it solves $\operatorname{Eexp}(-\xi X(t))=\exp (-t \Phi(\xi))$ for all $t, \xi \geq 0$. There are various relationships between the Laplace exponent $\Phi$ and the characteristic exponent $\Psi$. We mention one next: Let $\mathbf{S}:=\{S(t)\}_{t \geq 0}$ denote an independent symmetric Cauchy process [that is, $\mathbf{S}$ is a Lévy process with characteristic exponent $\Psi(\xi):=|\xi|$, so that $S(t) / t$ has the standard Cauchy distribution on the line for all $t>0]$ and note that the following from a few applications of Fubini's theorem: For all $t, \xi \geq 0$,

$$
\mathrm{e}^{-t \Phi(\xi)}=\mathrm{E}\left[\mathrm{e}^{-\xi X(t)}\right]=\mathrm{E}\left[\mathrm{e}^{i X(t) S(\xi)}\right]=\frac{1}{\pi} \int_{-\infty}^{\infty} \frac{\mathrm{e}^{-t \Psi(\xi z)}}{1+z^{2}} \mathrm{~d} z .
$$

We integrate both side $\left[\mathrm{e}^{-t} \mathrm{~d} t\right]$ to find that

$$
\frac{1}{1+\Phi(\xi)}=\frac{1}{\pi} W(1 / \xi) \quad \text { for all } \xi \geq 0 .
$$

Theorem 3.6 implies the following theorem of Horowitz [22]: With probability one,

$$
\operatorname{dim}_{\mathrm{H}} X[0, b]=\limsup _{\xi \rightarrow \infty} \frac{\log \Phi(\xi)}{\log \xi} .
$$

For an example, let us consider a one-dimensional Brownian motion $\mathbf{B}$; the theory of Brownian local times tells us that the zero set of $\mathbf{B}$ is the closure of the range 
of a subordinator $\mathbf{X}$ with $\Phi(\xi)=$ const $\cdot \xi^{1 / 2}$ for all positive $\xi$; see Maisonneuve [33], and also Bertoin [2, Chapter 9] for a pedagogic account. Because the closure of the range of $\mathbf{X}$ and the range itself differ in at most the jump points of $\mathbf{X}$ - and there are only countably-many of those - we can conclude the following well-known theorem of Lévy [31]: The Hausdorff dimension of the zero-set of Brownian motion is almost surely equal to $\lim \sup _{\xi \rightarrow \infty} \log \Phi(\xi) / \log \xi=1 / 2$. Lévy's theorem [for Brownian motion] has simpler proofs than the one outlined here. But the present method can be used to compute the Hausdorff dimension of the level sets of quite general Markov processes, when simpler arguments no longer exist.

\subsection{Proof of Theorem 3.1, and the odds of hitting a ball}

Define, for each $b \geq 0$, the incomplete renewal measure $U_{b}$ as follows:

$$
U_{b}(A):=\int_{0}^{b} \mathrm{P}\{X(s) \in A\} \mathrm{d} s .
$$

Each $U_{b}$ is a finite Borel measure on $\mathbf{R}^{d}$ of total mass $b$.

Define the ball

$$
B(x, r):=\left\{y \in \mathbf{R}^{d}:|x-y| \leq r\right\},
$$

where

$$
|z|:=\max _{1 \leq j \leq d}\left|z_{j}\right| \quad \text { for all } z \in \mathbf{R}^{d} .
$$

Then we have the following "quantitative hitting-time" estimate. The particular formulation that follows appeared in [27], but this is an old folklore result which arises in various forms in many parts of the literature.

Theorem 3.6. The following holds for all $x \in \mathbf{R}^{d}$ and $b, r>0$ :

$$
\frac{U_{b}(B(x, r))}{U_{b}(B(0,2 r))} \leq \mathrm{P}\{X[0, b] \cap B(x, r) \neq \varnothing\} \leq \frac{U_{2 b}(B(x, 2 r))}{U_{b}(B(0, r))} .
$$

Proof. Let $T$ denote the smallest time $s \in[0, b]$ at which $|X(s)-x| \leq r$; if such an $s$ does not exist, then set $T:=b+1$. We can write

$$
\begin{aligned}
U_{b}(B(x, r)) & =\mathrm{E}\left(\int_{T}^{b} \mathbf{1}_{\{|X(s)-x| \leq r\}} \mathrm{d} s ; 0 \leq T \leq b\right) \\
& =\mathrm{E}\left(\int_{0}^{b-T} \mathbf{1}_{\{|X(s+T)-X(T)+X(T)-x| \leq r\}} \mathrm{d} s ; 0 \leq T \leq b\right) \\
& \leq \mathrm{E}\left(\int_{0}^{b} \mathbf{1}_{\{|X(s+T)-X(T)+X(T)-x| \leq r\}} \mathrm{d} s ; 0 \leq T \leq b\right) .
\end{aligned}
$$


According to the strong markov property [3, Proposition 6, p. 20], the process $\{X(s+T)-X(T)\}_{s \geq 0}$ is a copy of $\mathbf{X}$, and is independent of $\{X(u)\}_{u \in[0, T]}$. It follows from this that

$$
\begin{aligned}
U_{b}(B(x, r)) & \leq \mathrm{E}\left[U_{b}(B(X(T)-x ; r)) ; 0 \leq T \leq b\right] \\
& \leq U_{b}(B(0,2 r)) \cdot \mathrm{P}\{0 \leq T \leq b\},
\end{aligned}
$$

because if $T \in[0, b]$ then $|X(T)-x| \leq r$, whence $B(X(T)-x, r) \subseteq B(0,2 r)$. This proves the first inequality of the theorem. The second inequality is proved similarly, but instead of the preceding with start with the following: For the same $T$ as before,

$$
\begin{aligned}
U_{2 b}(B(x, 2 r)) & \geq \mathrm{E}\left(\int_{T}^{2 b} \mathbf{1}_{\{|X(s)-x| \leq 2 r\}} \mathrm{d} s ; 0 \leq T \leq b\right) \\
& =\mathrm{E}\left(\int_{0}^{2 b-T} \mathbf{1}_{\{X(T+s)-X(T)+X(T)-x \mid \leq 2 r\}} \mathrm{d} s ; 0 \leq T \leq b\right) \\
& \geq \mathrm{E}\left(\int_{0}^{b} \mathbf{1}_{\{X(T+s)-X(T)+X(T)-x \mid \leq 2 r\}} \mathrm{d} s ; 0 \leq T \leq b\right) \\
& =\mathrm{E}\left(U_{b}(B(X(T)-x, 2 r)) ; 0 \leq T \leq b\right) .
\end{aligned}
$$

The theorem follows from this and an application of the triangle inequality; namely, that $B(X(T)-x, 2 r) \supseteq B(0, r)$ almost surely on $\{0 \leq T \leq b\}$.

Recall that the distribution of $X(s)$ is $\mu_{s}$. Thus, we define the renewal measure $U$ of the process $\mathbf{X}$ via

$$
U(A):=\int_{0}^{\infty} \mathrm{P}\{X(s) \in A\} \mathrm{e}^{-s} \mathrm{~d} s=\int_{0}^{\infty} \mu_{s}(A) \mathrm{e}^{-s} \mathrm{~d} s .
$$

Note that $U$ is a Borel probability measure on $\mathbf{R}^{d}$. Next we show that the complete renewal measure is estimated well by the incomplete ones.

Lemma 3.7. For all $b, r>0$,

$$
\mathrm{e}^{-b} U_{b}(B(0, r)) \leq U(B(0, r)) \leq \frac{16^{d}}{1-\mathrm{e}^{-b}} \cdot U_{b}(B(0, r)) .
$$

Proof. The first inequality is an elementary consequence of the definitions of $U$ and $U_{b}$; we derive the second one only. 
We can write

$$
\begin{aligned}
U(B(0, r)) & \leq \sum_{k=0}^{\infty} \mathrm{e}^{-k b} \int_{k b}^{(k+1) b} \mathrm{P}\{|X(s)| \leq r\} \mathrm{d} s \\
& =\sum_{k=0}^{\infty} \mathrm{e}^{-k b} \int_{0}^{b} \mathrm{P}\{|X(s+k b)-X(k b)+X(k b)| \leq r\} \mathrm{d} s \\
& =\sum_{k=0}^{\infty} \mathrm{e}^{-k b} \mathrm{E}\left[U_{b}(B(X(k b), r))\right],
\end{aligned}
$$

since $\{X(s+k b)-X(k b)\}_{s \geq 0}$ has the same distribution as the Lévy process $\mathbf{X}$, and is also independent of $X(k b)$.

Since probabilities are $\leq 1$, the first inequality in Theorem 3.6 tells us that

$$
\sup _{x \in \mathbf{R}^{d}} U_{b}(B(x, r)) \leq U_{b}(B(0,2 r)) \quad \text { for all } r>0 .
$$

Because we can cover $B(0,2 r)$ by at most $16^{d}$ disjoint balls of radius $(r / 2)$, the preceding shows that

$$
U_{b}(B(0,2 r)) \leq 16^{d} U(B(0, r)) .
$$

One more application of (3.19) shows that

$$
\sup _{x \in \mathbf{R}^{d}} U_{b}(B(x, r)) \leq 16^{d} U_{b}(B(0, r)) \quad \text { for all } r>0 .
$$

This and (3.18) together imply the second inequality of the lemma.

Proof of Theorem 3.1. Let $R(r)$ denote the $r$-enlargement of $-X[0, b]$ for every $r>0$. That is, $R(r):=\cup B(-X(s), r)$, where the union is taken over all $s \in[0, b]$. We might note that $\mathrm{P}\{X[0, b] \cap B(x, r) \neq \varnothing\}=\mathrm{E}\left[\mathbf{1}_{R(r)}(x)\right]$, and hence

$$
\int_{\mathbf{R}^{d}} \mathrm{P}\{X[0, b] \cap B(x, r) \neq \varnothing\} \mathrm{d} x=\mathrm{E}[\operatorname{leb}(R(r))],
$$

where "leb" denotes the Lebesgue measure on $\mathbf{R}^{d}$. We can integrate the inequalities of Theorem 3.6 with respect to $\mathrm{d} x$ to find that

$$
\mathrm{E}[\operatorname{leb}(R(r))] \asymp \frac{r^{d}}{U_{b}(B(0, r))},
$$

where $f \asymp g$ means that $(f / g)(r)$ is bounded away from zero and infinity by constants, uniformly over all $r>0$. [The preceding requires the Tonnelli theorem and the fact that the total mass of $U_{b}$ is finite and positive.]

Clearly, $\operatorname{leb}(R(r))$ converges downward to the Lebesgue measure of $X[0, b]$ as $r \downarrow 0$. Therefore, it suffices to prove that $\kappa$ is integrable iff $U_{b}(B(0, r))=O\left(r^{d}\right)$ as $r \downarrow 0$. Thanks to (3.20), it remains to prove the following:

$$
\kappa \in L^{1}\left(\mathbf{R}^{d}\right) \quad \text { iff } \quad U(B(0, r))=O\left(r^{d}\right) \text { as } r \downarrow 0 .
$$

We use Fourier analysis to establish this, and hence the theorem. 
Owing to (3.16), $\hat{U}(\xi)=\{1+\Psi(\xi)\}^{-1}$ defines the Fourier transform of $U$ in the sense of distributions; in particular, $|\hat{U}(\xi)| \leq 1$. Consequently, for all rapidlydecreasing test functions $\phi: \mathbf{R}^{d} \rightarrow \mathbf{R}$,

$$
\int \phi \mathrm{d} U=\frac{1}{(2 \pi)^{d}} \int_{\mathbf{R}^{d}} \operatorname{Re}\left(\frac{\overline{\hat{\phi}(\xi)}}{1+\Psi(\xi)}\right) \mathrm{d} \xi .
$$

Next we prove that the preceding holds for all uniformly continuous $\phi$ such that $\hat{\phi}$ is real and nonnegative. Indeed, let $\gamma_{n}$ denote the density function of the centered gaussian distribution on $\mathbf{R}^{d}$ whose covariance matrix is $1 / n$ times the identity, and then apply (3.25) to $\phi * \gamma_{n}$ in place of $\phi$ to find that

$$
\int\left(\phi * \gamma_{n}\right) \mathrm{d} U=\frac{1}{(2 \pi)^{d}} \int_{\mathbf{R}^{d}} \operatorname{Re}\left(\frac{\overline{\hat{\phi}(\xi)}}{1+\Psi(\xi)}\right) \mathrm{e}^{-\|\xi\|^{2} /(2 n)} \mathrm{d} \xi,
$$

for all $n \geq 1$. Because $\phi$ is uniformly continuous, $\phi * \gamma_{n} \rightarrow \phi$ uniformly as $n \rightarrow \infty$; and the left-hand side of (3.26) converges to $\int \phi \mathrm{d} U$ as $n \rightarrow \infty$. Because $\hat{\phi} \geq 0$, (3.25) follows from applying the monotone convergence theorem to the right-hand side of $(3.26)$.

Let $f_{r}(x):=(2 r)^{-d} \mathbf{1}_{B(0, r)}(x)$ and $\phi:=\phi_{r}:=f_{r} * f_{r}$. Since $\phi_{r}$ is uniformly continuous and $\hat{\phi}_{r}=\left|\hat{f}_{r}\right|^{2} \geq 0$, it follows that

$$
\int \phi_{r} \mathrm{~d} U=\frac{1}{(2 \pi)^{d}} \int_{\mathbf{R}^{d}} \hat{\phi}_{r}(\xi) \kappa(\xi) \mathrm{d} \xi .
$$

Because $2^{-d}(2 r)^{-d} \mathbf{1}_{B(0, r / 2)} \leq \phi_{r} \leq(2 r)^{-d} \mathbf{1}_{B(0,2 r)}$,

$$
\frac{U(B(0, r / 2))}{2^{d} \cdot(2 r)^{d}} \leq \int \phi_{r} \mathrm{~d} U \leq \frac{U(B(0,2 r)}{(2 r)^{d}} .
$$

Therefore, (3.24) is equivalent to the statement that $\kappa \in L^{1}\left(\mathbf{R}^{d}\right)$ iff $\int \phi_{r} \mathrm{~d} U=$ $O(1)$. Suppose, first, that $\kappa$ is integrable. Since $\left|\hat{\phi}_{r}\right| \leq 1$, (3.27) implies that $\lim \sup _{r \downarrow 0} \int \phi_{r} \mathrm{~d} U \leq(2 \pi)^{-d}\|\kappa\|_{L^{1}\left(\mathbf{R}^{d}\right)}<\infty$. For the converse, we merely observe that $(2 \pi)^{-d}\|\kappa\|_{L^{1}\left(\mathbf{R}^{d}\right)} \leq \liminf _{r \downarrow 0} \int \phi_{r} \mathrm{~d} U$, thanks to Fatou's lemma and the fact that $\lim _{r \downarrow 0} \hat{\phi}_{r}(\xi)=1$ for all $\xi$.

\subsection{A theorem of Pruitt, and proof of Theorem 3.4}

Theorem 3.8 (Pruitt [44]). For all $b>0$, the following holds almost surely:

$$
\operatorname{dim}_{\mathrm{H}} X[0, b]=\underline{\operatorname{dim}}_{\mathrm{M}} X[0, b]=\liminf _{r \downarrow 0} \frac{\log U(B(0, r))}{\log r}
$$

Proof. Let $N_{n}(b)$ denote the total number of dyadic cubes $I \in \mathcal{D}_{n}$ that intersect $X[0, b]$. According to Theorem 3.6,

$$
\mathrm{E}\left(N_{n}(b)\right) \leq \frac{1}{U_{b}\left(B\left(0,2^{-n-1}\right)\right)} \cdot \sum_{x: B\left(x, 2^{-n-1}\right) \in \mathcal{D}_{n}} U_{2 b}\left(B\left(x, 2^{-n}\right)\right) .
$$


Because of (3.19) and (3.20),

$$
\sup _{n \geq 1} \sum_{x: B\left(x, 2^{-n-1}\right) \in \mathcal{D}_{n}} U_{2 b}\left(B\left(x, 2^{-n}\right)\right)<\infty .
$$

Consequently,

$$
\mathrm{E}\left(N_{n}(b)\right) \leq \frac{\text { const }}{U\left(B\left(0,2^{-n}\right)\right)},
$$

thanks to Lemma 3.7 and (3.18). Let us select $q>0$ such that $U\left(B\left(0,2^{-n}\right)\right) \geq$ $2^{-n q}$ for infinitely-many $n$ tending to $\infty$. Then, (3.32) and Fatou's lemma together imply that $\liminf _{n \rightarrow \infty} 2^{-n q} N_{n}(b)<\infty$ almost surely. Therefore, $\operatorname{dim}_{\mathrm{H}} X[0, b] \leq$ $\operatorname{dim}_{\mathrm{M}} X[0, b] \leq q$ almost surely; this proves half of the theorem.

We prove the other half by appealing to Frostman's theorem (Theorem 1.1). Consider the following [random] Borel measure $\mu$ [compare with (2.8)]:

$$
\mu(V):=\int_{0}^{b} \mathbf{1}_{V}(X(s)) \mathrm{d} s .
$$

Then, $I_{q}(\mu)=\int_{[0, b]^{2}}|X(s)-X(t)|^{-q} \mathrm{~d} s \mathrm{~d} t$ for all $q>0$. We compute expectations.

$$
\mathrm{E}\left(I_{q}(\mu)\right)=2 \iint_{0 \leq s<t<b} \mathrm{E}\left(|X(t-s)|^{-q}\right) \mathrm{d} s \mathrm{~d} t \leq 2 b \int_{0}^{b} \mathrm{E}\left(|X(s)|^{-q}\right) \mathrm{d} s ;
$$

then we integrate by parts to find that

$$
\mathrm{E}\left(|X(s)|^{-q}\right)=q \int_{0}^{\infty} \lambda^{-q-1} \mathrm{P}\{|X(s)| \leq \lambda\} \mathrm{d} \lambda .
$$

It follows from the preceding and Lemma 3.7 that

$$
\begin{aligned}
\mathrm{E}\left(I_{q}(\mu)\right) & \leq 2 b q \int_{0}^{\infty} \lambda^{-q-1} U_{b}(B(0, \lambda)) \mathrm{d} \lambda \\
& \leq 2 b q \mathrm{e}^{b} \cdot \int_{0}^{\infty} \lambda^{-q-1} U(B(0, \lambda)) \mathrm{d} \lambda .
\end{aligned}
$$

If $q>0$ and $\epsilon>0$ are selected so that $U(B(0, r))=O\left(r^{q+\epsilon}\right)$ as $r \rightarrow 0$, then $I_{q}(\mu)$ is almost surely finite, since it will have a finite expectation. This proves the theorem.

Proof of Theorem 3.4. If $u \in L_{l o c}^{1}\left(\mathbf{R}^{d}\right)$, or $u$ is a locally finite Borel measure on $\mathbf{R}^{d}$, then $\hat{u}$ denotes its Fourier transform in the sense of Schwartz. We normalize the Fourier transform so that $\hat{u}(\xi)=\int_{\mathbf{R}^{d}} u(x) \exp (i \xi \cdot x) \mathrm{d} x$ whenever $u \in L^{1}\left(\mathbf{R}^{d}\right)$.

Consider the function

$$
\varphi_{r}(x):=\prod_{j=1}^{d}\left(\frac{1-\cos \left(2 r x_{j}\right)}{2 \pi r x_{j}^{2}}\right) \quad \text { for } x \in \mathbf{R}^{d},
$$


where $r>0$ is a parameter. Then $\varphi_{r}$ is a nonnegative integrable function on $\mathbf{R}^{d}$, and its Fourier transform is the Pólya kernel,

$$
\hat{\varphi}_{r}(\xi)=\prod_{j=1}^{d}\left(1-\frac{\left|\xi_{j}\right|}{2 r}\right)^{+}
$$

Note that $\hat{\varphi}_{r}(\xi) \geq 2^{-d}$ whenever $|\xi| \leq r$. That is,

$$
\mathbf{1}_{B(0, r)}(\xi) \leq 2^{d} \hat{\varphi}_{r}(\xi) \quad \text { for all } r>0 \text { and } \xi \in \mathbf{R}^{d} .
$$

Therefore, by Fubini's theorem,

$$
\mathrm{P}\{X(s) \in B(0, r)\} \leq 2^{d} \mathrm{E}\left(\hat{\varphi}_{r}(X(s))\right)=2^{d} \int_{\mathbf{R}^{d}} \hat{\varphi}_{r}(x) \mu_{s}(\mathrm{~d} x),
$$

where, we recall, $\mu_{s}:=\mathrm{P} \circ X(s)^{-1}$ denotes the distribution of $X(s)$. Consequently,

$$
\mathrm{P}\{X(s) \in B(0, r)\} \leq 2^{d} \int_{\mathbf{R}^{d}} \varphi_{r}(\xi) \hat{\mu}_{s}(\xi) \mathrm{d} \xi=2^{d} \int_{\mathbf{R}^{d}} \varphi_{r}(\xi) e^{-s \Psi(\xi)} \mathrm{d} \xi .
$$

We integrate $\left[\mathrm{e}^{-s} \mathrm{~d} s\right]$ to find that

$$
U(B(0, r)) \leq 2^{d} \int_{\mathbf{R}^{d}} \varphi_{r}(\xi) \kappa(\xi) \mathrm{d} \xi ;
$$

only the real part [part of the definition of $\kappa$ ] enters because $U(B(0, r))$ and $\phi_{r}(\xi)$ are real valued. Since $(1-\cos z) / z^{2} \leq$ const $/\left(1+z^{2}\right)$ for all real numbers $z$, this proves that $U(B(0, r)) \leq$ const $\cdot W(r)$, whence half of the theorem.

For the other inequality we choose and fix $\delta \in(0,1)$, and note that for every $r>0$ and $z \in \mathbf{R}^{d}$,

$$
\mathbf{1}_{B(0, r)}(z) \geq \exp \left(-\frac{1}{r^{1-\delta}} \sum_{j=1}^{d}\left|z_{j}\right|\right)-\exp \left(-r^{-1+\delta}\right) .
$$

Plug in $z:=X(s)$, take expectations, and then integrate $\left[\mathrm{e}^{-s} \mathrm{~d} s\right]$ to find that

$$
U(B(0, r)) \geq \mathrm{E}\left[\int_{0}^{\infty} \exp \left(-\frac{1}{r^{1-\delta}} \sum_{j=1}^{d}\left|X_{j}(s)\right|\right) \mathrm{e}^{-s} \mathrm{~d} s\right]-\exp \left(-r^{-1+\delta}\right) .
$$

But with probability one,

$$
\exp \left(-\frac{1}{r^{1-\delta}} \sum_{j=1}^{d}\left|X_{j}(s)\right|\right)=\mathrm{E}\left[\exp \left(i \frac{S \cdot X(s)}{r^{1-\delta}}\right) \mid X(s)\right],
$$

where $S:=\left(S_{1}, \ldots, S_{d}\right)$ is a vector of $d$ independent standard-Cauchy random variables; the probability density function of $S$ is $p(\xi):=\pi^{-d} \prod_{j=1}^{d}\left(1+\xi_{j}^{2}\right)^{-1}$ at 
$\xi \in \mathbf{R}^{d}$. By Fubini's theorem, and after a calculation, we find that

$$
\begin{aligned}
U(B(0, r)) & \geq \mathrm{E}\left[\int_{0}^{\infty} \exp \left(i \frac{S \cdot X(s)}{r^{1-\delta}}\right) \mathrm{e}^{-s} \mathrm{~d} s\right]-\exp \left(-r^{-1+\delta}\right) \\
& =\int_{0}^{\infty} \mathrm{e}^{-s} \mathrm{~d} s \int_{\mathbf{R}^{d}} p(\xi) \mathrm{d} \xi \mathrm{E}\left[\exp \left(i \frac{\xi \cdot X(s)}{r^{1-\delta}}\right)\right]-\exp \left(-r^{-1+\delta}\right) \\
& =\frac{1}{\pi^{d}} W\left(r^{1-\delta}\right)-\exp \left(-r^{-1+\delta}\right)
\end{aligned}
$$

And this is sufficient to prove the remaining direction of the theorem.

\subsection{Occupation measures, local times, and Hawkes's theorem}

Let $\mathbf{X}$ denote a Lévy process on $\mathbf{R}^{d}$, and define

$$
Q(G):=\int_{0}^{\infty} \mathbf{1}_{G}(X(s)) \mathrm{e}^{-s} \mathrm{~d} s,
$$

for all Borel sets $G \subseteq \mathbf{R}^{d}$. Evidently, $Q$ is a [random] Borel probability measure on $\mathbf{R}^{d}$. We follow Geman and Horowitz [17], and say that $\mathbf{X}$ has square-integrable local times if $Q$ is absolutely continuous with respect to leb, and its Radon-Nikodým density $\ell$ satisfies $\ell:=\mathrm{d} Q / \mathrm{d} x \in L^{2}\left(\mathbf{R}^{d}\right)$. The random process $\{\ell(x)\}_{x \in \mathbf{R}^{d}}$ is then called the local times of $\mathbf{X}$. Note that if $\mathbf{X}$ has square-integrable local times, then the following holds: For all nonrandom Borel-measurable functions $f: \mathbf{R}^{d} \rightarrow \mathbf{R}_{+}$,

$$
\int_{0}^{\infty} f(X(s)) \mathrm{e}^{-s} \mathrm{~d} s=\int_{\mathbf{R}^{d}} f(x) \ell(x) \mathrm{d} x \quad \text { almost surely. }
$$

In words, local times exist iff $Q$ is differentiable. In this way, local times are the most natural "Frostman-like" measures that can be constructed on the range of a given Lévy process. These local times will make a surprising appearance in the following section on stochastic PDEs, as well.

Theorem 3.9 (Hawkes [20]). A Lévy process $\mathbf{X}$ has square-integrable local times if and only if the Lebesgue measure of $X\left(\mathbf{R}_{+}\right)$is positive with positive probability. Another equivalent condition is that $\kappa \in L^{1}\left(\mathbf{R}^{d}\right)$.

Proof. Theorem 3.1 shows the equivalence of the assertion " $\kappa \in L^{1}\left(\mathbf{R}^{d}\right)$ " and the statement "leb $\left(X\left(\mathbf{R}_{+}\right)\right)>0$ with positive probability."

If $\ell$ exists and is almost surely in $L^{2}\left(\mathbf{R}^{d}\right)$, then we can apply (3.48) to deduce that $\ell$ is a random probability density on the closure of the range of $\mathbf{X}$. In particular, the closure of $\mathbf{X}$ - and hence $\mathbf{X}$ itself - must have positive Lebesgue measure almost surely.

Conversely, suppose the Lebesgue measure of $X\left(\mathbf{R}_{+}\right)$is positive with positive probability. Equivalently, that $\kappa \in L^{1}\left(\mathbf{R}^{d}\right)$. I will follow Kahane [24], and use Fourier analysis to show that $\ell$ exists and is in $L^{2}\left(\mathbf{R}^{d}\right)$ almost surely. 
Because $\hat{Q}(\xi)=\int_{0}^{\infty} \exp \{-s+i \xi \cdot X(s)\} \mathrm{d} s$ for all $\xi \in \mathbf{R}^{d}$, we can write $\mathrm{E}\left(|\hat{Q}(\xi)|^{2}\right)$ as $T_{1}+T_{2}$, where

$$
\begin{aligned}
& T_{1}:=\iint_{0<s<t<\infty} \mathrm{e}^{-s-t} \mathrm{E}\left(\mathrm{e}^{i \xi \cdot[X(s)-X(t)]}\right) \mathrm{d} s \mathrm{~d} t, \\
& T_{2}:=\iint_{0<t<s<\infty} \mathrm{e}^{-s-t} \mathrm{E}\left(\mathrm{e}^{i \xi \cdot[X(s)-X(t)]}\right) \mathrm{d} s \mathrm{~d} t .
\end{aligned}
$$

If $s<t$, then the distribution of $X(s)-X(t)$ is the same as that of $-X(t-s)$, and hence

$$
\begin{aligned}
T_{1} & =\iint_{0<s<t<\infty} \mathrm{e}^{-s-t} \mathrm{E}\left(\mathrm{e}^{-i \xi \cdot X(t-s)}\right) \mathrm{d} s \mathrm{~d} t \\
& =\iint_{0<s<t<\infty} \mathrm{e}^{-s-t} \mathrm{e}^{-(t-s) \overline{\Psi(\xi)}} \mathrm{d} s \mathrm{~d} t=\frac{1}{2+2 \overline{\Psi(\xi)}} .
\end{aligned}
$$

Similarly, $T_{2}=\{2+2 \Psi(\xi)\}^{-1}$, and hence,

$$
\mathrm{E}\left(|\hat{Q}(\xi)|^{2}\right)=\operatorname{Re}\left(\frac{1}{1+\Psi(\xi)}\right)=\kappa(\xi) .
$$

Because we have assumed that $\kappa$ is integrable on $\mathbf{R}^{d}$, this proves that $\hat{Q} \in L^{2}\left(\mathbf{R}^{d}\right)$ almost surely. Plancherel's theorem ensures us that $Q$ is almost surely absolutely continuous with respect to the Lebesgue measure, and has an almost-surely squareintegrable density $\ell$.

\subsection{The sum of the range of a Lévy process and a set}

Let $G$ denote a fixed Borel-measurable subset of $\mathbf{R}^{d}$, and $\mathbf{X}$ a Lévy process on $\mathbf{R}^{d}$. We wish to know when $X\left(\mathbf{R}_{+}\right) \oplus G$ has positive $d$-dimensional Lebesgue measure [with positive probability], where $A \oplus B:=\{a+b: a \in A, b \in B\}$. There are good reasons for asking such a question. For instance, if we consider $G:=\{0\}$, then this is asking for when the range of $X$ has positive measure; and the answer is given by Theorem 3.1 in this case. Or if $\mathbf{X}$ is a "nice" Lévy process - such as the Brownian motion - then our question turns out to be equivalent to asking when $\mathrm{P}\left\{0 \in X\left(\mathbf{R}_{+}\right) \oplus G\right\}>0$. If we can answer this for all Borel sets $G$, then by conditioning we can decide when $\mathrm{P}\left\{X\left(\mathbf{R}_{+}\right) \cap Y\left(\mathbf{R}_{+}\right) \neq \varnothing\right\}>0$ where $Y$ is an independent "nice" Lévy process on $\mathbf{R}^{d}$. That is, we can decide when the trajectories of two independent Lévy processes can intersect. There are many other applications of these ideas as well.

Theorem 3.10 (Hawkes [21]). Let $\mathbf{X}$ denote a Lévy process on $\mathbf{R}^{d}$ and $G \subset \mathbf{R}^{d}$ a nonrandom Borel-measurable set. Then the Lebesgue measure of $X\left(\mathbf{R}_{+}\right) \oplus G$ is 
positive with positive probability iff there exists a compactly supported probability measure $\nu$ on $G$ such that

$$
\int_{\mathbf{R}^{d}} \kappa(\xi)|\hat{\nu}(\xi)|^{2} \mathrm{~d} \xi<\infty
$$

where $\kappa$ was defined in (3.2).

The method of proof implies the following quantitative improvement:

$$
\begin{aligned}
\sup _{b>0} \mathrm{e}^{-b} \mathrm{E}[\operatorname{leb}(X[0, b] \oplus G)] \leq\left[\frac{1}{(2 \pi)^{d}} \inf _{\nu \in \mathcal{P}(G)} \int_{\mathbf{R}^{d}} \kappa(\xi)|\hat{\nu}(\xi)|^{2} \mathrm{~d} \xi\right]^{-1} & \leq \mathrm{E}\left[\operatorname{leb}\left(X\left(\mathbf{R}_{+}\right) \oplus G\right)\right],
\end{aligned}
$$

where inf $\varnothing:=\infty$, and $1 / \infty:=0$. Clearly, Theorem 3.10 is a consequence of $(3.52)$.

Example. Condition (3.52) is frequently a fractal and/or capacity condition on $G$. For instance, consider the case that $\mathbf{X}$ is an isotropic stable process with index $\alpha \in(0,2]$. That is, $\Psi(\xi):=$ const. $\|\xi\|^{\alpha}$; when $\alpha=2$ this means that $\mathbf{X}$ is Brownian motion. One can easily check that (3.52) holds if and only if $\int_{\mathbf{R}^{d}}\|\xi\|^{-\alpha}|\hat{\nu}(\xi)|^{2} \mathrm{~d} \xi<$ $\infty$. Thus, a little Fourier analysis [50, Theorem 5, p. 73] shows that, in the present setting, (3.52) is equivalent to the condition that $I_{d-\alpha}(\nu)<\infty$ for some $\nu \in \mathcal{P}(G)$, where $I_{q}(\nu)$ is the same Riesz energy that was defined earlier in (1.8). In particular, Frostman's theorem (Theorem 1.1) implies that, in this example, $X\left(\mathbf{R}_{+}\right) \oplus G$ can have positive Lebesgue measure if $\operatorname{dim}_{\mathrm{H}} G>d-\alpha$, but not if $\operatorname{dim}_{\mathrm{H}} G<d-\alpha$. This finding is essentially due to McKean [37].

The most natural proof of Theorem 3.10 requires developing too much analytic/probabilistic machinery. Instead I will prove a close variant which has fewer requirements [though it does assume a good knowledge of abstract harmonic analysis at the level of Loomis [32], Pontryagin [42], or Rudin [47].]

Let $\Gamma$ denote a separable compact metric abelian group, metrizable by a distance $d$ which is compatible with the group structure of $\Gamma$. As is customary for abelian groups, we denote the identity of $\Gamma$ by " 0 ," the inverse of $g \in \Gamma$ by $-g$, and group multiplication by "+." We denote the Haar measure on $\Gamma$ by $m$, using the standard normalization, $m(\Gamma)=1[32,42,47]$.

Let $\mathbf{Y}:=\{Y(t)\}_{t \geq 0}$ be a Lévy process with values on $\Gamma$. That is:

1. $Y(0)=0$ almost surely;

2. $Y(t+s)-Y(s)$ is independent of $\{Y(u)\}_{0 \leq u \leq s}$ for all $s, t \geq 0$;

3. The distribution of $Y(t+s)-Y(s)$ does not depend on $s$, for all $s, t \geq 0$; and

4. $t \mapsto Y(t)$ is continuous in probability; i.e., $\lim _{s \rightarrow t} \mathrm{P}\{d(X(s), X(t))>\epsilon\}=0$ for all $t \geq 0$ and $\epsilon>0$.

Define $\Gamma^{*}$ to be the dual group to $\Gamma$; every character $\xi \in \Gamma^{*}$ can be identified with a one-to-one continuous mapping from $\Gamma$ onto the unit disc in $\mathbf{C}$ such that $\xi(x+y)=\xi(x) \xi(y)$. It is well known that because $\Gamma$ is compact, $\Gamma^{*}$ is discrete/countable. The distribution of the entire process $\mathbf{Y}$ is determined uniquely 
by a function $\psi: \Gamma^{*} \rightarrow \mathbf{C}$ that satisfies the following:

$$
\mathrm{E}[\xi(Y(t))]=\exp (-t \psi(\xi)) \quad \text { for all } t \geq 0 \text { and } \xi \in \Gamma^{*} .
$$

We call $\psi$ the characteristic exponent of $\mathbf{Y}$.

For all intents and purposes, you might wish to consider only the case that $\Gamma$ is the torus $(0,2 \pi]^{d}$, in which case $\Gamma^{*}:=\mathbf{Z}^{d}$ and $\xi(x)=\exp (i \xi \cdot x)$ for all $x \in \Gamma$ and $\xi \in \Gamma^{*}$. Then we have the following variant of Theorem 3.10:

Theorem 3.11. Let $G \subset \Gamma$ be a nonrandom Borel-measurable set. Then the Haar measure of $Y\left(\mathbf{R}_{+}\right) \oplus G$ is positive with positive probability if and only if there exists a compactly-supported probability measure $\nu$ on $G$ such that

$$
\sum_{\xi \in \Gamma^{*}} K(\xi)|\hat{\nu}(\xi)|^{2}<\infty
$$

where

$$
K(\xi):=\operatorname{Re}\left(\frac{1}{1+\psi(\xi)}\right) \quad \text { for all } \xi \in \Gamma^{*}
$$

In fact, I will establish the following analogue of (3.52):

$$
\begin{aligned}
\sup _{b>0} \mathrm{e}^{-b} \mathrm{E}[m(Y[0, b] \oplus G)] \leq\left[\inf _{\mathcal{P}(G)} \sum_{\xi \in \Gamma^{*}} K(\xi)|\hat{\nu}(\xi)|^{2}\right]^{-1} & \leq \mathrm{E}\left[m\left(Y\left(\mathbf{R}_{+}\right) \oplus G\right)\right] ;
\end{aligned}
$$

which appears to be a new result with novel ideas of proof. I will not prove Theorem 3.10 here. But suffice it to say that one can deduce Theorem 3.10 from Theorem 3.11 - which I will prove - upon first letting $\Gamma$ be the large torus $[0,2 \pi n)^{d}$, and then "letting $n \uparrow \infty$." There are other ways of proceeding, as well.

As first step, let us recall a classical inequality.

Lemma 3.12 (Paley-Zygmund [40]). If $X \in L^{2}(\mathrm{P})$ is nonzero with positive probability, then for all $\lambda \in[0,1]$,

$$
\mathrm{P}\{X \geq \lambda \mathrm{E} X\} \geq \frac{(1-\lambda)^{2}(\mathrm{E} X)^{2}}{\mathrm{E}\left(X^{2}\right)} .
$$

Proof. If $A:=\{X \geq \lambda \mathrm{E} X\}$, then

$$
\mathrm{E} X=\mathrm{E}\left(X \mathbf{1}_{A}\right)+\mathrm{E}\left(X \mathbf{1}_{A^{c}}\right) \leq \sqrt{\mathrm{E}\left(X^{2}\right) \cdot \mathrm{P}(A)}+\lambda \mathrm{E} X,
$$

by the Cauchy-Schwarz inequality. Solve for $\mathrm{P}(A)$ to finish.

Now we can proceed with the bulk of the argument. 
Proof of Theorem 3.11. It suffices to prove this theorem in the case that $G$ is closed. We assume this condition on $G$ henceforth.

Let $\mathrm{P}_{a}$ denote the distribution of the process $a+\mathbf{X}$ for all $a \in \Gamma$, and let $\mathrm{E}_{a}$ denote the corresponding expectation operator. We will be working with the probability measure $\mathrm{P}_{m}:=\int_{\Gamma} m(\mathrm{~d} a) \mathrm{P}_{a}$ and its expectation operator $\mathrm{E}_{m}:=$ $\int_{\Gamma} m(\mathrm{~d} a) \mathrm{E}_{a}$.

Suppose $h$ is a probability density on $\Gamma$, and consider

$$
J(h):=\int_{0}^{\infty} h(-X(s)) \mathrm{e}^{-s} \mathrm{~d} s .
$$

Since $\mathrm{E}_{a}[J(h)]=\int_{0}^{\infty} \mathrm{E}[h(a-X(s))] \mathrm{e}^{-s} \mathrm{~d} s$, we integrate $[m(\mathrm{~d} a)]$ to find that

$$
\mathrm{E}_{m}[J(h)]=\int h(x) m(\mathrm{~d} x) \cdot \int_{0}^{\infty} \mathrm{e}^{-s} \mathrm{~d} s=1 .
$$

Similarly, we can compute directly to find that

$$
\mathrm{E}_{m}\left(|J(h)|^{2}\right)=2 \iint_{0<s<t<\infty} \mathrm{e}^{-s-t} \mathrm{~d} s \mathrm{~d} t \int_{\Gamma} m(\mathrm{~d} b) h(b) \mathrm{E}[h(b-X(t-s))] .
$$

Since the distribution of $X(t-s)$ is $\mu_{t-s}$, it follows that $\mathrm{E}[h(b-X(t-s))]=$ $\left(\mu_{t-s} * h\right)(b)$, where "*" denotes convolution on the group algebra. After an appeal or two to the Tonnelli theorem we find that

$$
\mathrm{E}_{m}\left(|J(h)|^{2}\right)=\int_{\Gamma} h(b)(U * h)(b) m(\mathrm{~d} b)
$$

where $U$ is the renewal measure from (3.16). If, in addition, $h \in L^{2}(\Gamma)$, then $U * h \in L^{2}(\Gamma)$ also, and hence by Plancherel's theorem,

$$
\mathrm{E}_{m}\left(|J(h)|^{2}\right)=\sum_{\xi \in \Gamma^{*}} \operatorname{Re} \hat{U}(\xi)|\hat{h}(\xi)|^{2}
$$

Because $\hat{\mu}_{t}(\xi)=\exp (-t \psi(\xi))$, it follows that $\operatorname{Re} \hat{U}(\xi)=K(\xi)$, whence

$$
\mathrm{E}_{m}\left(|J(h)|^{2}\right)=\sum_{\xi \in \Gamma^{*}} K(\xi)|\hat{h}(\xi)|^{2}
$$

This, (3.61) and Lemma 3.12 together imply that

$$
\mathrm{P}_{m}\{J(h)>0\} \geq \frac{1}{\sum_{\xi \in \Gamma^{*}} K(\xi)|\hat{h}(\xi)|^{2}} .
$$

Now consider a function $h$ of the form $h(x):=\left(\nu * \phi_{\epsilon}\right)(x)$, where: (i) $\nu \in \mathcal{P}(G)$; and (ii) $\left\{\phi_{\epsilon}\right\}_{\epsilon>0}$ is a continuous [compactly-supported] approximation to the identity. If $J\left(\nu * \phi_{\epsilon}\right)>0$, then certainly $-X(s) \in G^{\epsilon}$ for some $s>0$, where $G^{\epsilon}$ denotes the $\epsilon$-enlargement of $G$. Since $|\hat{h}(\xi)| \leq|\hat{\nu}(\xi)|$, we obtain the following after we let $\epsilon \downarrow 0$ :

$$
\mathrm{P}_{m}\left\{\overline{-Y\left(\mathbf{R}_{+}\right)} \cap G \neq \varnothing\right\} \geq\left[\inf _{\nu \in \mathcal{P}(G)} \sum_{\xi \in \Gamma^{*}} K(\xi)|\hat{\nu}(\xi)|^{2}\right]^{-1} .
$$


This proves the first inequality in (3.53), since we can let $\epsilon \downarrow 0$ in the following:

$$
\begin{aligned}
\mathrm{P}_{m}\left\{-Y\left(\mathbf{R}_{+}\right) \cap G^{\epsilon} \neq \varnothing\right\} & =\int_{\Gamma} \mathrm{P}\left\{a-Y(s) \in G^{\epsilon} \text { for some } s>0\right\} m(\mathrm{~d} a) \\
& =\mathrm{E}\left[m\left(Y\left(\mathbf{R}_{+}\right) \oplus G^{\epsilon}\right)\right] .
\end{aligned}
$$

Now we strive to establish the second inequality in (3.53). Without loss of generality, we may assume that $\mathrm{E}[m(Y[0, b] \oplus G)]>0$; for there is nothing left to prove otherwise.

In order to obtain the converse we need some jargon from stochastic analysis. Let $\mathcal{F}:=\left\{\mathcal{F}_{t}\right\}_{t \geq 0}$ denote the filtration generated by the process $\mathbf{Y}$; we can and will assume, without any loss in generality, that $\mathcal{F}$ satisfies the "usual conditions" [10], so that in particular Doob's optional stopping theorem applies.

Let $T$ be the first hitting time of $-G$. That is,

$$
T:=\inf \{s>0:-Y(s) \in G\},
$$

where $\inf \varnothing:=\infty$, as before. Then $T$ is a stopping time with respect to $\mathcal{F}$. For all density functions $h: \Gamma \rightarrow \mathbf{R}_{+}$,

$$
\begin{aligned}
\mathrm{E}_{m}\left(J(h) \mid \mathcal{F}_{T}\right) & \geq \mathrm{E}_{m}\left(\int_{T}^{\infty} h(Y(s)) \mathrm{e}^{-s} \mathrm{~d} s \mid \mathcal{F}_{T}\right) \cdot \mathbf{1}_{\{T<\infty\}} \\
& =\mathrm{e}^{-T} \mathrm{E}_{m}\left(\int_{0}^{\infty} h(Y(s+T)) \mathrm{e}^{-s} \mathrm{~d} s \mid \mathcal{F}_{T}\right) \cdot \mathbf{1}_{\{T<b\}}
\end{aligned}
$$

We apply the strong markov property at time $T$ to find that

$$
\mathrm{E}_{m}\left[h(Y(s+T)) \mid \mathcal{F}_{T}\right]=\left(\mu_{s} * h\right)(Y(T)) \quad \text { almost surely on }\{T<\infty\},
$$

where $\mu_{s}$ denotes the distribution of $Y(s)$ now. Consequently,

$$
\mathrm{E}_{m}\left(J(h) \mid \mathcal{F}_{T}\right) \geq \mathrm{e}^{-b}(U * h)(Y(T)) \cdot \mathbf{1}_{\{T<b\}},
$$

where $U$ is the renewal measure, defined by (3.16). Since $\mathrm{E}_{m}(J(h))=1$, an appeal to Doob's optional stopping theorem yields the following:

$$
1 \geq \mathrm{e}^{-b} \mathrm{E}[(U * h)(Y(T)) \mid T<b] \cdot \mathrm{P}_{m}\{T<b\} .
$$

Let $\rho(\bullet):=\mathrm{P}_{m}\{Y(T) \in \bullet \mid T<b\}$. Thus, we have

$$
\mathrm{E}[m(Y[0, b] \oplus G)]=\mathrm{P}_{m}\{T<b\} \leq \frac{\mathrm{e}^{b}}{\int(U * h) \mathrm{d} \rho} .
$$

[The identity follows as in (3.68).] Since $U$ is a probability measure on $\Gamma$, if $h \in L^{2}(\Gamma)$, then we can apply Plancherel's theorem to find that $\int_{\Gamma}(U * h) \mathrm{d} \rho=$ $\sum_{\xi \in \Gamma^{*}} \hat{U}(\xi) \hat{h}(\xi) \overline{\hat{\rho}(\xi)}$, and hence

$$
\mathrm{E}[m(Y[0, b] \oplus G)] \leq \frac{\mathrm{e}^{b}}{\operatorname{Re} \sum_{\xi \in \Gamma^{*}} \hat{U}(\xi) \hat{h}(\xi) \overline{\hat{\rho}(\xi)}} .
$$

Since $\Gamma$ is compact, the preceding holds for all continuous functions $h$, for example. Now consider $h:=\breve{\rho} * \phi_{\epsilon} * \breve{\phi}_{\epsilon}$, where: (i) $\breve{f}(x):=f(-x)$ for all functions $f: \Gamma \rightarrow \mathbf{R}$ and $x \in \Gamma$; (ii) $\breve{\rho}(A):=\rho(-A)$ for all Borel sets $A \subset \Gamma$; and (iii) $\left\{\phi_{\epsilon}\right\}_{\epsilon>0}$ is 
an approximation to the identity comprised of all continuous functions. Thus, we obtain

$$
\begin{aligned}
\mathrm{E}[m(Y[0, b] \oplus G)] & \leq \frac{\mathrm{e}^{b}}{\sum_{\xi \in \Gamma^{*}} \operatorname{Re} \hat{U}(\xi) \cdot\left|\hat{\phi}_{\epsilon}(\xi)\right|^{2} \cdot|\hat{\rho}(\xi)|^{2}} \\
& =\frac{\mathrm{e}^{b}}{\sum_{\xi \in \Gamma^{*}} K(\xi) \cdot\left|\hat{\phi}_{\epsilon}(\xi)\right|^{2} \cdot|\hat{\rho}(\xi)|^{2}} .
\end{aligned}
$$

The second inequality in (3.53) follows from the preceding, and Fatou's lemma, upon letting $\epsilon \downarrow 0$.

\section{Linear Stochastic PDEs}

Let that $\mu_{s}$ denotes the distribution of a Lévy process $\mathbf{X}$ on $\mathbf{R}^{d}$. We have noted already that $\mu_{s+t}=\mu_{s} * \mu_{t}$, and therefore we can view $\left\{\mu_{t}\right\}_{t \geq 0}$ as a convolution semigroup of linear operators acting on $L^{2}\left(\mathbf{R}^{d}\right)$.

Define

$$
\operatorname{Dom}[\mathcal{L}]:=\left\{f \in L_{l o c}^{1}\left(\mathbf{R}^{d}\right): \int_{\mathbf{R}^{d}}|\hat{f}(\xi)|^{2}\left(1+|\Psi(\xi)|^{2}\right) \mathrm{d} \xi<\infty\right\} .
$$

If $f \in \operatorname{Dom}[\mathcal{L}]$, then the dominated convergence theorem shows that for all $g \in$ $L^{2}\left(\mathbf{R}^{d}\right)$ the following limit exists, and the ensuing computation is valid:

$$
\begin{aligned}
\int_{\mathbf{R}^{d}} g(x)(\mathcal{L} f)(x) \mathrm{d} x & :=\lim _{s \downarrow 0} \int_{\mathbf{R}^{d}} g(x)\left[\frac{\left(\mu_{s} * f\right)(x)-f(x)}{s}\right] \mathrm{d} x \\
& =\lim _{s \downarrow 0} \frac{1}{(2 \pi)^{d}} \int_{\mathbf{R}^{d}} \overline{\hat{g}(\xi)} \hat{f}(\xi)\left[\frac{\mathrm{e}^{-s \Psi(\xi)}-1}{s}\right] \mathrm{d} \xi \\
& =-\int_{\mathbf{R}^{d}} \overline{\hat{g}(\xi)} \hat{f}(\xi) \Psi(\xi) \mathrm{d} \xi
\end{aligned}
$$

Owing to duality, this defines a linear operator $\mathcal{L}$, mapping $\operatorname{Dom}[\mathcal{L}]$ in to $L^{2}\left(\mathbf{R}^{d}\right)$, such that: (1) $\mathcal{L}$ is the Hille-Yosida generator of $\left\{\mu_{s}\right\}_{s \geq 0}$ in the $L^{2}$-sense; and (2) the Fourier multiplier of $\mathcal{L}$ is $-\Psi$.

Example. I mention a few examples:

1. If $\Psi(\xi)=c\|\xi\|^{\alpha}$ for some $c>0$ and $\alpha \in(0,2]$, then $\mathbf{X}$ is an isotropic stable process on $\mathbf{R}^{d}$ with index $\alpha$-Brownian motion if $\alpha=2$ - and $\mathcal{L}=c \Delta^{\alpha / 2}$ is $c$ times the fractional Laplacian of order $\alpha / 2$.

2. When $d=1$ and $\mathbf{X}$ is a Poisson process on $\mathbf{Z}_{+}:=\{0,1,2, \ldots\}$ with rate $\lambda>$ 0 , then we have $\Psi(\xi)=\lambda\left(1-\mathrm{e}^{i \xi}\right)$, and $(\mathcal{L} f)(x)=\lambda\{f(x)-f(x-1)\} \mathbf{1}_{\mathbf{z}_{+}}(x)$ is $\lambda$ times the discrete gradient on $\mathbf{Z}_{+}$.

3. When $d=1$ and $\mathbf{X}$ is a compensated Poisson process on $\mathbf{R}$ with rate $\lambda$, $\Psi(\xi)=\lambda\left(1+i \xi-\mathrm{e}^{i \xi}\right)$, and $(\mathcal{L} f)(x)=\lambda\left\{f(x)-f(x-1)-f^{\prime}(x)\right\}$ for all $x \in \mathbf{R}$.

For more information see Fukushima et al [16] and Jacob [23]. 
The object of interest here is the so-called stochastic heat equation,

$$
\frac{\partial u(t, x)}{\partial t}=(\mathcal{L} u)(t, x)+\dot{W}(t, x),
$$

where $\mathcal{L}$ is the $L^{2}$-generator of a Lévy process $\mathbf{X}$ on $\mathbf{R}^{d}$, and $\dot{W}$ denotes white noise. That is,

$$
\dot{W}(t, x):=\frac{\partial^{d+1} W(t, x)}{\partial t \partial x_{1} \cdots \partial x_{d}}
$$

in the sense of generalized random fields, where $\mathbf{W}:=\{W(t, x)\}_{(t, x) \in \mathbf{R}^{d+1}}$ is Brownian sheet with $d+1$ parameters. That is, $\mathbf{W}$ is a continuous centered Gaussian random field with the following covariance: For all $(t, x),(s, y) \in \mathbf{R}^{d+1}$,

$$
\operatorname{Cov}(W(t, x), W(s, y))=\min (s, t) \cdot \prod_{j=1}^{d} \min \left(x_{j}, y_{j}\right) .
$$

There are many ways to make rigorous sense of the stochastic heat equation (4.3). Here is a quick, though perhaps not the most informative, way: Let $\phi: \mathbf{R}^{d} \rightarrow$ $\mathbf{R}$ be a smooth compactly-supported function from $\mathbf{R}^{d}$ to $\mathbf{R}$. We can multiply both sides of (4.3), purely formally, by $\phi(x)$ and integrate $[\mathrm{d} x]$ to arrive at the "equation,"

$$
\frac{\partial u(t, \phi)}{\partial t}=(\mathcal{L} u)(t, \phi)+\dot{W}(t, \phi),
$$

where $g(t, \phi):=\int_{\mathbf{R}^{d}} g(t, x) \phi(x) \mathrm{d} x$, whenever this makes sense, and $\dot{W}(t, \phi) \mathrm{d} t=$ $\mathrm{d} X_{t}$ - as generalized Gaussian random fields - where $X$ is a Brownian motion with covariance function

$$
\mathrm{E}\left(X_{s} X_{t}\right)=\|\phi\|_{L^{2}\left(\mathbf{R}^{d}\right)}^{2} \cdot \min (s, t) .
$$

It is now possible to convince oneself that (4.6) ought to be interpreted as an infinite family of correlated stochastic differential equations, one for each nice $\phi$. If the ensuing solution $u(t, \phi)$ can indeed be written as $\int_{\mathbf{R}^{d}} u(t, x) \phi(x) \mathrm{d} x$, then $\{u(t, x)\}_{t>0, x \in \mathbf{R}^{d}}$ is a "random-field solution." References [6, 8, 25, 29, 30, 43, 46, $52]$ contain ways of interpreting (4.3) and many other stochastic PDEs.

We will interpret (4.3) Fourier-analytically, and prove the following:

Theorem 4.1 (Dalang [9], Khoshnevisan, Foondun, and Nualart [14]). Let $\mathbf{X}^{\prime}$ denote an independent copy of $\mathbf{X}$, and consider the Lévy process $\mathbf{Y}:=\{Y(t)\}_{t \geq 0}$ where $Y(t):=X(t)-X^{\prime}(t)$ for all $t \geq 0$. Then, (4.3) has a random-field solution $\{u(t, x)\}_{t \geq 0, x \in \mathbf{R}^{d}}$ iff the range of $\mathbf{Y}$ has square-integrable local times $\{\ell(x)\}_{x \in \mathbf{R}^{d}}$.

In particular, (4.3) never has random-field solutions when $d \geq 2$ (Remark $3.2)$.

We prove Theorem 4.1 after we discuss the meaning of (4.3) in detail; we shall see that the proof is based on simple ideas. Let us also mention the following deeper result whose proof is too difficult to be included here.

Theorem 4.2 (Khoshnevisan, Foondun, and Nualart [14]). Suppose $d=1$ and (4.3)

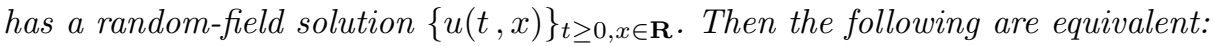


- $x \mapsto u(t, x)$ is continuous with positive probability for some $t>0$;

- $x \mapsto u(t, x)$ is continuous almost surely for some $t>0$;

- $x \mapsto u(t, x)$ is continuous almost surely for all $t>0$;

- $x \mapsto \ell(x)$ is almost surely continuous.

The preceding continues to hold if "continuous" is replaced by "Hölder continuous" everywhere, and the critical index of Hölder continuity of $x \mapsto u(t, x)$ is the same as that of $x \mapsto \ell(x)$.

There is a large literature that describes the continuity of local times of Lévy processes. This literature culminates in the work of Barlow [1]. In order to describe that work let us consider the following function:

$$
\delta(x, y):=\int_{-\infty}^{\infty} \frac{1-\cos ((x-y) \xi)}{1+2 \operatorname{Re} \Psi(\xi)} \mathrm{d} \xi .
$$

We can compare Theorem 4.1 with Theorem 3.9 to see that (4.3) has random-field solutions if and only if $\delta(x, x)<\infty$ for all $x$. It follows easily from this that $\delta$ is a [pseudo-] metric on $\mathbf{R}$. Define $N_{\delta}(\epsilon)$ to be the smallest number of $\delta$-balls of radius $\epsilon>0$ that are needed to cover $[0,1] ; N_{\delta}$ is the so-called Kolmogorov metric entropy of $[0,1] .{ }^{6}$ Then we have

Theorem 4.3 (Barlow [1]). Suppose the symmetrized Lévy process $\mathbf{Y}$ has squareintegrable local times $\{\ell(x)\}_{x \in \mathbf{R}}$. Then $x \mapsto \ell(x)$ is continuous almost surely if and only if it is continuous with positive probability. And the following is a necessary and sufficient condition for that continuity:

$$
\int_{0^{+}}\left(\log N_{\delta}(\epsilon)\right)^{1 / 2} \mathrm{~d} \epsilon<\infty .
$$

Barlow's theorem provides an analytic condition for the continuity of $x \mapsto$ $\ell(x)$. According to Theorem 4.2, the same condition is necessary and sufficient for the continuity of the solution to (4.3) in its space variable $x$. In light of footnote 6 , eq. (4.9) is a non-euclidean fractal-like condition, on the psuedo-metric space $([0,1], \delta)$, that is necessary and sufficient for the continuity of solutions to the stochastic heat equation. [In fact, we shall see that there is basically only one good solution to (4.3) if there are any.] We close this article by making sense of (4.3) and subsequently proving Theorem 4.1.

\subsection{White noise and Wiener integrals}

Let $\mathcal{T}_{d}:=[0, \infty) \times \mathbf{R}^{d}$ denote "space-time." We write a typical element of $\mathcal{T}_{d}$ as $(t, x)$ where $t \geq 0$ and $x \in \mathbf{R}^{d}$.

Consider the standard [complex] Hilbert space $H:=L^{2}\left(\mathcal{T}_{d}\right)$, endowed with the usual inner product $\langle g, h\rangle_{H}:=\int_{0}^{\infty} \mathrm{d} t \int_{\mathbf{R}^{d}} \mathrm{~d} x g(t, x) \overline{h(t, x)}$ and norm $\|h\|_{H}:=$

\footnotetext{
${ }^{6}$ We can replace $([0,1], \mathrm{d})$ by a general compact metric space $(K, \mathrm{~d})$. And $N_{\mathrm{d}}$ is defined in the same way. If we apply it with a compact set $K \subset \mathbf{R}^{d}$ where $\mathrm{d}:=$ the usual euclidean metric instead of the preceding one, then $\lim \sup _{\epsilon \downarrow} \log N_{\delta}(\epsilon) / \log (1 / \epsilon)=\overline{\operatorname{dim}}_{\mathrm{M}} K$.
} 
$\langle h, h\rangle_{H}^{1 / 2}$ for all $g, h \in H$. The isonormal process $\mathbf{W}:=\{W(h)\}_{h \in H}$ is a [complexvalued] mean-zero gaussian process whose covariance function is described by

$$
\operatorname{Cov}(W(h), W(g)):=\langle h, g\rangle_{H} .
$$

It is not difficult to prove that for all $a, b \in \mathbf{C}$ and $h, g \in H$ the following holds almost surely: $W(a h+b g)=a W(h)+b W(g)$. But the null set depends on $a, b, h$, and $g$. In particular, if $\operatorname{Im} f \equiv 0$ then $W(f)$ is real-valued, and the restriction of $\mathbf{W}$ to such functions is a real-valued isonormal process.

For all nonrandom Borel sets $A \subset \mathcal{T}_{d}$ with finite Lebesgue measure define

$$
\dot{W}(A):=W\left(\mathbf{1}_{A}\right) .
$$

The resulting set-indexed stochastic process $\dot{\mathbf{W}}$ is called white noise on $\mathcal{T}_{d}$. Thus, we can think of $h \mapsto W(h)$ as an integral against white noise, and write

$$
W(h)=\int_{0}^{\infty} \int_{\mathbf{R}^{d}} h(t, x) \dot{W}(\mathrm{~d} t \mathrm{~d} x) \quad \text { for all } h \in H .
$$

This is called the Wiener integral of $h$. We will stop writing the dot in $\dot{W}$ from here on, as there is no ambiguity in omitting that dot. Thus, from now on, we write

$$
W(h):=\int_{0}^{\infty} \int_{\mathbf{R}^{d}} h(t, x) W(\mathrm{~d} t \mathrm{~d} x) \quad \text { for all } h \in H .
$$

\subsection{The Fourier transform of white noise}

If $h \in H$, then so is $\hat{h}$, where $\hat{h}(t, \xi):=\int_{\mathbf{R}^{d}} \mathrm{e}^{i \xi \cdot x} h(t, x) \mathrm{d} x$ for all $\xi \in \mathbf{R}^{d} ; \hat{h}$ is well defined for almost all $t \geq 0$. And we can define the Fourier transform $\hat{W}$ of white noise as

$$
\hat{W}(h):=W(\hat{h}) .
$$

Thus, $\{\hat{W}(h)\}_{h \in H}$ is a mean-zero [complex-valued] gaussian random field whose covariance function is defined by

$$
\operatorname{Cov}(\hat{W}(f), \hat{W}(g))=\langle\hat{f}, \hat{g}\rangle_{H}=(2 \pi)^{d}\langle f, g\rangle_{H} ;
$$

the last assertion follows from Plancherel's theorem when $f(t, x)$ is of the form $T(t) X(x)$, and in general by density.

\subsection{A return to the linear stochastic heat equation}

Before we make rigorous sense of (4.3), let us recall a few facts about the linear heat equation of classical PDEs.

Suppose $w(t, x)$ defines a "nice" function, and consider the heat equation

$$
\frac{\partial u(t, x)}{\partial t}=(\mathcal{L} u)(t, x)+w(t, x) .
$$


We can make sense of (4.16) by taking the Fourier transform [in $x$ ] throughout. This yields $\partial \hat{u}(t, \xi) / \partial t=-\Psi(\xi) \hat{u}(t, \xi)+\hat{w}(t, \xi)$. Thus, a reasonable solution $u$ to (4.16) ought to satisfy

$$
\hat{u}(t, \xi)=\int_{0}^{t} \mathrm{e}^{-(t-s) \Psi(\xi)} \hat{w}(s, \xi) \mathrm{d} s .
$$

In particular, we might expect that if $\phi: \mathbf{R}^{d} \rightarrow \mathbf{R}$ is also "nice," then

$$
\int_{\mathbf{R}^{d}} \hat{u}(t, \xi) \overline{\hat{\phi}(\xi)} \mathrm{d} \xi=\int_{0}^{t} \mathrm{~d} s \int_{\mathbf{R}^{d}} \mathrm{~d} \xi \overline{\hat{\phi}(\xi)} \mathrm{e}^{-(t-s) \Psi(\xi)} \hat{w}(s, \xi) .
$$

An informal appeal to the Plancherel theorem might then suggest that

$$
u(t, \phi)=\frac{1}{(2 \pi)^{d}} \int_{0}^{t} \mathrm{~d} s \int_{\mathbf{R}^{d}} \mathrm{~d} \xi \bar{\phi}(\xi) \mathrm{e}^{-(t-s) \Psi(\xi)} \hat{w}(s, \xi),
$$

where $u(t, \phi):=\int_{\mathbf{R}^{d}} u(t, x) \phi(x) \mathrm{d} x$. A remarkable feature of this heuristic computation is that it produces the usual notion of weak solutions of (4.16) rigorously, for instance when $\phi$ is in the Wiener algebra $L^{1}\left(\mathbf{R}^{d}\right) \cap \mathcal{F}^{-1}\left(L^{1}\left(\mathbf{R}^{d}\right)\right)$, where $\mathcal{F}$ denotes the Fourier transform.

Let $\mathfrak{D}(\Psi)$ denote the class of Schwartz distributions $\phi$ on $\mathbf{R}^{d}$ whose Fourier transform $\hat{\phi}$ is a function and

$$
\int_{0}^{t} \mathrm{~d} s \int_{\mathbf{R}^{d}} \mathrm{~d} \xi\left|\hat{\phi}(\xi) \mathrm{e}^{-s \Psi(\xi)}\right|^{2}<\infty
$$

see Dalang [9]. Then we say that $\mathfrak{D}(\Psi)$ is the class of natural testing distributions for (4.3), and the weak solution $\{u(t, \phi)\}_{\phi \in \mathfrak{D}(\Psi)}$ to (4.3) is the random field [i.e., stochastic process] defined by the resulting Wiener integrals

$$
u(t, \phi):=\frac{1}{(2 \pi)^{d}} \int_{0}^{t} \int_{\mathbf{R}^{d}} \overline{\hat{\phi}(\xi)} \mathrm{e}^{-(t-s) \Psi(\xi)} \hat{W}(\mathrm{~d} s \mathrm{~d} \xi) .
$$

This is a well-defined random field, indexed by $t \geq 0$ and $\phi \in \mathfrak{D}(\Psi)$, because

$$
\mathrm{E}\left(|u(t, \phi)|^{2}\right)=\frac{1}{(2 \pi)^{d}} \int_{0}^{t} \mathrm{~d} s \int_{\mathbf{R}^{d}} \mathrm{~d} \xi\left|\hat{\phi}(\xi) \mathrm{e}^{-(t-s) \Psi(\xi)}\right|^{2}<\infty .
$$

Moreover, one can show that our "weak solution" $u(t, \phi)$ agrees almost surely with the much-better known "weak solution" of Walsh [52] for all $\phi \in L^{2}\left(\mathbf{R}^{d}\right)$. [We will not dwell on this connection here.]

Definition 4.4. We say that (4.3) has a random-field solution $\{u(t, x)\}_{t \geq 0, x \in \mathbf{R}^{d}}$ if and only if $\delta_{x} \in \mathfrak{D}(\Psi)$ for one, and hence, all $x \in \mathbf{R}^{d}$. In that case, we identify $u(t, x)$ with $u\left(t, \delta_{x}\right)$ for each $t \geq 0$ and $x \in \mathbf{R}^{d}$.

This is consistent with its analogue in PDEs. In fact, if $u(t, x)$ exists and is sufficiently regular, then $\int_{\mathbf{R}^{d}} u(t, x) \phi(x) \mathrm{d} x$ defines a version of $u(t, \phi)$.

Define

$$
\mathcal{E}(\phi, \psi):=\frac{1}{(2 \pi)^{d}} \int_{\mathbf{R}^{d}} \frac{\hat{\psi}(\xi) \overline{\hat{\phi}(\xi)}}{1+2 \operatorname{Re} \Psi(\xi)} \mathrm{d} \xi,
$$


for all Schwartz distributions $\phi$ and $\psi$ whose Fourier transform is a function and $\mathcal{E}(\phi, \phi)+\mathcal{E}(\psi, \psi)<\infty$. Because the real part of $\Psi$ is nonnegative, $\mathcal{E}(\phi, \phi)<\infty$ for all $\phi \in L^{2}\left(\mathbf{R}^{d}\right)$.

Lemma 4.5. For all $t \geq 0$ and $\phi \in L^{2}\left(\mathbf{R}^{d}\right)$,

$$
\left(1-\mathrm{e}^{-t}\right) \mathcal{E}(\phi, \phi) \leq \mathrm{E}\left(|u(t, \phi)|^{2}\right) \leq \mathrm{e}^{t} \mathcal{E}(\phi, \phi) .
$$

In particular, $L^{2}\left(\mathbf{R}^{d}\right) \subseteq \mathfrak{D}(\Psi)$.

Proof. Note that $|\exp \{-s \Psi(\xi)\}|^{2}=\exp \{-2 s \operatorname{Re} \Psi(\xi)\}$ and $\operatorname{Re} \Psi(\xi) \geq 0$. Thus, for all $t>0$ and $\phi \in \mathfrak{D}(\Psi)$,

$$
\mathrm{E}\left(|u(t, \phi)|^{2}\right)=\frac{1}{(2 \pi)^{d}} \int_{0}^{t} \mathrm{~d} s \int_{\mathbf{R}^{d}} \mathrm{~d} \xi|\hat{\phi}(\xi)|^{2} \mathrm{e}^{-2 s \operatorname{Re} \Psi(\xi)} .
$$

For all $\phi \in L^{2}\left(\mathbf{R}^{d}\right)$,

$$
\mathrm{E}\left(|u(t, \phi)|^{2}\right) \leq \frac{\mathrm{e}^{t}}{(2 \pi)^{d}} \int_{0}^{\infty} \mathrm{d} s \int_{\mathbf{R}^{d}} \mathrm{~d} \xi|\hat{\phi}(\xi)|^{2} \mathrm{e}^{-s-2 s \operatorname{Re} \Psi(\xi)}=\mathrm{e}^{t} \mathcal{E}(\phi, \phi) .
$$

In order to derive the complementary bound we note that

$$
\begin{aligned}
\frac{1}{1+2 \operatorname{Re} \Psi(\xi)} & =\sum_{n=0}^{\infty} \int_{n t}^{(n+1) t} \mathrm{e}^{-s-2 s \operatorname{Re} \Psi(\xi)} \mathrm{d} s \\
& \leq \sum_{n=0}^{\infty} \mathrm{e}^{-n t} \int_{n t}^{(n+1) t} \mathrm{e}^{-2 s \operatorname{Re} \Psi(\xi)} \mathrm{d} s \\
& \leq \frac{1}{1-\mathrm{e}^{-t}} \cdot \int_{0}^{t} \mathrm{e}^{-2 r \operatorname{Re} \Psi(\xi)} \mathrm{d} r \quad[r:=s-n t] .
\end{aligned}
$$

The lemma follows from this and an application of (4.25).

Let us conclude the paper by proving Theorem 4.1.

Proof of Theorem 4.1. Lemma 4.5 identifies $\mathfrak{D}(\Psi)$ with the closure of $L^{2}\left(\mathbf{R}^{d}\right)$ in the "energy norm," $\phi \mapsto \mathcal{E}(\phi, \phi)^{1 / 2}$. In particular, we have a random-field solution if and only if $\mathcal{E}\left(\delta_{x}, \delta_{x}\right)<\infty$. An equivalent condition is the integrability of the function $\{1+2 \operatorname{Re} \Psi\}^{-1}$ on $\mathbf{R}^{d}$. Since $2 \operatorname{Re} \Psi$ is the characteristic exponent of the symmetrized Lévy process $\mathbf{Y}$, Hawkes's theorem (Theorem 3.9) completes the proof.

\section{Acknowledgments}

I would like to thank Professors Bandt, Mörters, and Zähle for encouraging me to write an exposition of this material. This material is based in part on a decade or two of collaborations with Professor Yimin Xiao. I thank him wholeheartedly for that, and for his suggestions and corrections to the present paper. Final thanks are owed to Dr. Michael Hinz, who has kindly provided me with a long list of corrections, together with many excellent suggestions for stylistic improvements. 


\section{References}

[1] M. T. Barlow, Necessary and sufficient conditions for the continuity of local time of Lévy processes, Ann. Probab. 16, No. 4 (1988) 1389-1427.

[2] J. Bertoin, Subordinators, in: Lecture Notes in Mathematics 1717, 1-91, SpringerVerlag, Berlin, 1999.

[3] J. Bertoin, Lévy Processes, Cambridge University Press, Cambridge, 1998.

[4] S. Bochner, Harmonic Analysis and the Theory of Probability, University of California Press, Berkeley and Los Angeles, 1955.

[5] É. Borel, Les probabilités dénombrables et leurs applications arithmétiques, Supplemento di rend. circ. Mat. Palermo 27 (1909) 247-271.

[6] P.-L. Chow, Stochastic Partial Differential Equations, Chapman \& Hall/CRC, Boca Raton, Fl., 2007.

[7] M. Csörgö and P. Révész, Strong Approximations in Probability and Statistics, Academic Press, N.Y., 1981.

[8] G. Da Prato and J. Zabczyk, Stochastic Equations in Infinite Dimensions, Cambridge University Press, Cambridge, 1992.

[9] R. C. Dalang, Extending the martingale measure stochastic integral with applications to spatially homogeneous s.p.d.e.'s, Electron. J. Probab. 4 (1999) no. 6, 29 pp. (electronic).

[10] C. Dellacherie and P.-A. Meyer, Probabilities and Potential B, translated from the French by J. P. Wilson, North-Holland Publishing Co., Amsterdam, 1982.

[11] H. G. Eggleston, The fractional dimension of a set defined by decimal properties, Quart. J. Math. (1949) Oxford Ser. 20, 31-36.

[12] K. J. Falconer, Fractal Geometry, second edition, Wiley, New York, 2003.

[13] K. J. Falconer, Random fractals, Math. Proc. Camb. Phil. Soc. 100 (1986) no. 3, 559-582.

[14] M. Foondun, D. Khoshnevisan, and E. Nualart, A local-time correspondence for stochastic partial differential equations (2008) preprint.

[15] O. Frostman, Potentiel d'équilibre et capacité des ensembles avec quelques applications à la théorie des fonctions, Meddel. Lunds. Univ. Mat. Sem. 3 (1935) 1-118.

[16] M., Fukushima, Y. Ōshima, and M. Takeda, Dirichlet Forms and Symmetric Markov Processes, Walter de Gruyter \& Co., Berlin, 1994.

[17] D. Geman and J. Horowitz, Occupation densities, Ann. Probab. 8 (1980) no. 1, 1-67.

[18] G. Grimmett, Random flows: network flows and electrical flows through random media, in: Surveys in Combinatorics, 1985 (Glasgow, 1985), 59-95, London Math. Soc. Lecture Note Ser. 103 Cambridge University Press, Cambridge, 1985.

[19] F. Hausdorff, Grundzüge der Mengerlehre, Verlag von Veit \& Comp., Leipzig, 1914.

[20] J. Hawkes, Local times as stationary processes, in: "From Local Times to Global Geometry, Control, and Physics" (Coventry, 1984/85), 111-120, Pitman Lecture Notes, Math. Ser. 150, Longman Science Tech., Harlow, 1986.

[21] J. Hawkes, Potential theory of Lévy processes, Proc. London Math. Soc. 38 (1979) 335-352. 
[22] J. Horowitz, The Hausdorff dimension of the sample path of a subordinator, Israel J. Math. 6 (1968) 176-182.

[23] N. Jacob, Pseudo Differential Operators and Markov Processes. Vol. I, Imperial College Press, London, 2001.

[24] J.-P. Kahane, Some Random Series of Functions, second edition, Cambridge University Press, Cambridge, 1985.

[25] G. Kallianpur and J. Xiong, Stochastic Differential Equations in Infinite Dimensional Spaces, Institute of Math. Statist. Lecture Notes-Monograph Series, Hayward, California, 1995.

[26] H. Kesten, Hitting Probabilities of Single Points for Processes with Stationary Independent Increments. Memoirs of the Amer. Math. Soc. 93, Amer. Math. Soc., Providence, R.I., 1969.

[27] D. Khoshnevisan, Escape rates for Lévy processes, Stud. Sci. Math. Hung. 33 (1997) 177-183.

[28] D. Khoshnevisan and Y. Xiao, Packing dimension of the range of a Lévy process, Proc. Amer. Math. Soc. 136 (2008) no. 7, 2597-2607.

[29] P. Kotelenez, Stochastic Ordinary and Stochastic Partial Differential Equations, Springer, New York, 2008.

[30] N. V. Krylov, On the foundations of the $L_{p}$-theory of stochastic partial differential equations, in: Stochastic Partial Differential Equations and Applications-VII, 179191, Lecture Notes in Pure \& Appl. Math., Chapman \& Hall/CRC, Boca Raton, Fl., 2006.

[31] P. Lévy, Processus Stochastiques et Mouvement Brownien, Gauthier-Villars, Paris, 1948.

[32] L. H. Loomis, Harmonic Analysis, Notes by Ethan Bolker, John W. Blattner, and Sylvan Wallach. 1965 MAA Cooperative Summer Seminar Math. Assoc. of America, Buffalo, N.Y., 1965.

[33] B. Maisonneuve, Ensembles régénératifs, temps locaux et subordinateurs, in: Lecture Notes in Math. 1527, 111-235, Springer, Berlin, 1971.

[34] B. B. Mandelbrot, The Fractal Geometry of Nature, W. H. Freeman \& Company, San Francisco, 1982.

[35] P. Mattila, Geometry of Sets and Measures in Euclidean Spaces, Cambridge University Press, Cambridge, 1995.

[36] R. D. Mauldin and S. C. Williams, S. C., Random recursive constructions: asymptotic geometric and topological properties, Trans. Amer. Math. Soc. 295 (1986) no. 1, 325346.

[37] H. McKean, Jr., Hausdorff-Besicovitch dimension of Brownian motion paths, Duke Math. J. 22 (1955) 229-234.

[38] S. Orey, Polar sets for processes with stationary independent increments, in: Markov Processes and Potential Theory (Proc. Sympos. Math. Res. Center, Madison, Wis., 1967), 117-126, Wiley, New York, 1967.

[39] Orey, S. and W. E. Pruitt, Sample functions of the N-parameter Wiener process, Ann. Probab. 1 (1973), 138-163. 
[40] R. E. A. C. Paley and A. Zygmund, A note on analytic functions in the unit circle, Proc. Camb. Phil. Soc. 28 (1932) 266-272.

[41] Y. Peres, Probability on Trees, in: Lecture Notes in Mathematics 1717, 195-280, Springer-Verlag, Berlin, 1999.

[42] L. S. Pontryagin, Topological Groups, Translated from the second Russian edition by Arlen Brown, Gordon and Breach Science Publishers, Inc., New York, 1966.

[43] C. Prévôt and M. Röckner, A Concise Course on Stochastic Partial Differential Equations, Lecture Notes in Mathematics 1905, Springer-Verlag, Berlin, Heidelberg, 2007.

[44] W. E. Pruitt, The Hausdorff dimension of the range of a process with stationary independent increments, J. Math. Mech. 19 (1969) 71-378.

[45] C. A. Rogers and S. J. Taylor, Functions continuous and singular with respect to a Hausdorff measure, Mathematika 8 (1961) 1-3.

[46] B. L. Rozovskiǔ, Stochastic Evolution Systems, Kluwer Academic Publishing Group, Dordrecht (Translated from the original Russian by A. Yarkho, Math. and Its Applicatons (Soviet Series), 35), 1990.

[47] W. Rudin, Fourier Analysis on Groups, Reprint of the 1962 original, John Wiley \& Sons, Inc., New York, 1990.

[48] K.-I. Sato, Lévy Processes and Infinitely Divisible Distributions, Cambridge University Press, Cambridge [translated from the 1990 Japanese original, revised by the author],1999.

[49] I. J. Schoenberg, Metric spaces and positive definite functions. Trans. Amer. Math. Soc. 44 (1938) no. 3, 522-536.

[50] E. Stein, Singular Integrals and Differentiability Properties of Functions, Fifth printing with corrections from the original in 1970, Princeton University Press, Princeton, N.J., 1986.

[51] S. J. Taylor, The Hausdorff $\alpha$-dimensional measure of Brownian paths in n-space, Proc. Camb. Phil. Soc. 49 (1953) 31-39.

[52] J. B. Walsh, An Introduction to Stochastic Partial Differential Equations, in: École d'été de probabilités de Saint-Flour, XIV-1984, Lecture Notes in Math. 1180, 265439, Springer, Berlin, 1986.

Davar Khoshnevisan

University of Utah

Department of Mathematics

155 South 1400 East JWB 233

Salt Lake City UT 84112-0090

U.S.A.

e-mail: davar@math.utah.edu 